\title{
On the role of fibre bonds on the elasticity of low-density papers: a micro-mechanical approach
}

\author{
L. Orgéas ${ }^{1}$, P.J.J. Dumont, , F. Martö̈a ${ }^{2}$, C. Marulier ${ }^{1,3}$, S. Le Corre L $^{4}$ D. Caillerie $^{1}$ \\ ${ }^{1}$ Univ. Grenoble Alpes, CNRS, Grenoble INP, 3SR Lab, F-38000 Grenoble, France \\ ${ }^{2}$ Univ. Lyon, CNRS, INSA-Lyon, LaMCoS, F-69621 Lyon, France \\ ${ }^{3}$ Univ. Grenoble Alpes, CNRS, Grenoble INP, LGP2, F-38000 Grenoble, France \\ ${ }^{4}$ Univ. Nantes, CNRS, Polytech Nantes, LTN, F-44000 Nantes, France \\ ${ }^{*}$ Corresponding author's email: laurent.orgeas@3sr-grenoble.fr
}

\begin{abstract}
Fine prediction of the elastic properties of paper materials can now be obtained using sophisticated fibre scale numerical approaches. However, there is still a need, in particular for low-density papers, for simple and compact analytical models that enable the elastic properties of these papers to be estimated from the knowledge of various structural information about their fibres and their fibrous networks. For that purpose, we pursued the analysis carried out in (Marulier et al., Cellulose, 22 (2015) 1517-39) with low-density papers that were fabricated with planar random and orientated fibrous microstructures and different fibre contents. The fibrous microstructures of these papers were imaged using X-ray synchrotron microtomography. The corresponding 3D images revealed highly connected fibrous networks with small fibre bond areas. Furthermore, the evolutions of their Young's moduli were nonlinear and evolved as power-laws with the fibre content. Current analytical models of the literature do not capture these trends. In light of these experimental data, we developed a fibre network model for the in-plane elasticity of papers in which the main deformation mechanisms of the micromechanical model is the shear of the numerous fibre bonds and their vicinity, whereas the fibre parts far from these zones were considered as rigid bodies. The stiffness tensor of papers was then estimated both numerically using a discrete element code and analytically using additional assumptions. Both approaches nicely fit the experimental trends by adjusting a unique unknown micromechanical parameter, which is the shear stiffness of bonding zones. The estimate of this parameter is relevant in light of several recently reported experimental results.
\end{abstract}

KEYWORDS: paper mechanics, X-ray microtomography, fibre bonds, fibre network model.

\section{1 - Introduction}

Paper is one of the most widespread man-made bio-based fibrous materials that is used in many engineering fields including packaging (Twede et al., 2014) (Viguié et al., 2011), health and medical care (Oyola-Reynoso et al., 2015), energy (Jabbour et al., 2013), filtration, fluid purification (Macfarlane et al., 2012), building or other structural applications (Martoïa et al., 
2017). The main constituents of paper are lignocellulosic fibres (mainly extracted from wood) that are strongly linked together by cohesive bonds (Hirn and Schennach 2015) in the form of fibrous networks with planar fibre orientation (Niskanen 2011) (Alava and Niskanen 2006). Papers with a wide diversity of physical and mechanical properties can be fabricated by tailoring the nature of the raw materials and the papermaking conditions (e.g., type and geometry of fibres, refining, forming, pressing and drying conditions, adding mineral fillers and other chemical additives...). However, the links between the papermaking parameters, the resulting fibrous microstructures and the product performances are still not fully evidenced. For example, the Young's modulus of low-density papers often follows a non-linear evolution with their density (Rigdahl et al., 1983, Niskanen 2011, Park et al., 2020, Wu and Dzenis 2005), the microstructural and micromechanical origins of which are still questioning. Thus, building the aforementioned links constitutes a subject of intense research. In particular, there is still a need for relevant multiscale mechanical constitutive laws that could (i) account for the complexity of paper microstructures and related deformation mechanisms and (ii) be implemented in numerical simulation tools (Simon 2020) for computer-aided design of paper applications or for monitoring of the papermaking process itself.

For that purpose, numerous theoretical studies have been conducted during the last decades. In most cases, paper materials are modelled as planar networks of deformable elastic fibres (Cox 1952) (Page and Seth 1980) (Schulgasser 1981) (Schulgasser and Page 1988) (Åström et al., 2000) (Alava and Niskanen 2006) (Godinho et al., 2019). These approaches often assume that the stiffness of the fibre bonds is relatively high (or infinite) so that the stretching, bending and shear of fibres far from bonds are the governing deformation mechanisms. The proposed analytical models are interesting because they clearly emphasize the role played by the content, geometry and mechanics of fibres. However, they do not account for the complexity of the geometry and micro-mechanics of fibre bonds and their close vicinity.

To better understand the effects of deformation micro-mechanisms on the mechanics of papers, many fibre scale numerical studies were conducted (Räisänen et al., 1997) (Åström et al., 2000) (Heyden 2000) (Wu and Dzenis, 2005) (Kulachenko and Uesaka 2012) (Kulachenko et al., 2012) (Mansour et al., 2019) (Motamedian et al., 2019) (Brandberg and Kulachenko 2020). For instance, Heyden et al. performed numerical simulations using networks of elastic beams to investigate the effects of the volume fraction, orientation, length, curl, and stiffness of fibres on the in-plane stiffness of low-density papers (Heyden and Gustafsson 1998) (Heyden 2000). Similarly, using 2D network simulation, Wu and Dzenis (2005) highlighted the pronounced non-linear evolution of the Young's modulus with the fibre content of low-density papers with planar random orientation. These analyses were recently extended for denser papers (Kulachenko et al., 2012) (Motamedian et al., 2019) (Brandberg and Kulachenko 2020) (Borodulina et al., 2018) (Brandberg and Kulachenko 2017) (Motamedian and Kulachenko 2019). Some of these studies showed that the geometry and the mechanics of fibre bonds could significantly contribute to the macroscale mechanical response of papers (Borodulina et al., 2018) (Brandberg and Kulachenko 2017) (Motamedian and Kulachenko 2019). In particular, for low-density fibrous networks made of stiff elastic fibres connected with sufficiently soft elastic bonds, Brandberg and Kulachenko (2017) showed that the contribution of the elastic energy of bonds to the overall elastic energy of fibrous networks was predominant compared with the other contributions related to the fibre deformation modes (bending, shear, tension). 
Bearing in mind that the volume fraction of bonding zones is much smaller than the volume fraction of the rest of the fibrous network, these results demonstrate that the intrinsic elastic energy stored in bonds is much higher than the intrinsic energy stored elsewhere. Thus, these numerical results confirm the trends reported in several complementary experimental studies (Page et al., 1962) (Page et al., 1979) (Page and Seth 1980) (Nanko and Ohsawa 1989) (DeMaio et al., 2006) (DeMaio and Patterson 2008) (Magnusson 2016) and highlight (i) the central role of fibre bond zones (the bonds and their vicinity) and (ii) the need of a proper characterisation of the geometry and micro-mechanics of fibre bonds. This is particularly true for low-density papers (Brandberg and Kulachenko, 2017).

On the one hand, noticeable progress has been achieved purposely to characterise the mechanics of individual fibre bonds thanks to micro-mechanical testing devices (Fischer et al., 2012) (Schmied et al., 2012) (Jajcinovic et al., 2016). These tests are interesting but still remain difficult to carry out as they require the isolation of the bonds and well-controlled constraints to avoid any deformation of the two bonded fibres. On the other hand, significant progress has also been realised to finely characterise the paper microstructures with $3 \mathrm{D}$ imaging techniques such as microtome serial sectioning (Kappel et al., 2009) or X-ray microtomography (Gregersen et al., 2001) (Roscoat et al., 2005) (Rolland du Roscoat et al., 2007) (Marulier et al., 2012) (Marulier et al., 2015) coupled with dedicated image analysis procedures (Viguié et al., 2013) (Wernersson et al., 2014) (Borodulina 2016) (Krasnoshlyk et al. 2018). For instance, we investigated the effect of forming, pressing and drying conditions on several microstructure descriptors of low-density papers from high resolution 3D images obtained with X-ray synchroton microtomography (Marulier et al., 2012) (Marulier et al., 2015). Unprecedented 3D description of the geometries of individual fibres and fibre bonds were obtained and discussed. However, we did not fully analyse these interesting 3D geometrical data, in particular concerning the geometry of fibre bonds, and did not take this information into account in a multiscale mechanical model to predict the mechanical properties of papers.

Thus, the objective of this study is to propose multiscale mechanical models for the elasticity of low-density paper materials, taking into account the descriptors of the microstructure that can be obtained using in-depth characterisation of 3D images. For that purpose, two types of low-density papers with in-plane isotropic and orientated fibrous microstructures were fabricated using unbeaten bleached and classified pulp fibres. Their microstructures were characterised using X-ray synchrotron microtomography (Marulier et al., 2015) and we herein completed the analysis of the 3D images acquired previously. We also characterised their tensile elastic properties. Combining these data allowed relevant assumptions for the architecture of fibre networks and their fibre scale mechanics to be stated. Then, these features were upscaled using the homogenisation with multiple scale asymptotic expansions for discrete structures (Tollenaere and Caillerie 1998) (Le Corre et al., 2004). This method proposes a rigorous framework to deduce the constitutive properties and the balance equations of the papers' equivalent continuum media. It also provides well-posed localisation problems to be solved on representative elementary volumes (REVs) to estimate their macroscale properties. These problems were first solved numerically using the discrete element method. Then, a second analytical multiscale model was proposed using additional simplifying assumptions. Predictions of the numerical and analytical models were compared with experimental data and discussed. 


\section{2 - Materials and experimental methods}

\subsection{Pulp fibres and related low-density papers}

Unbeaten bleached and classified softwood pulp fibres from maritime pine were used to fabricate papers using the procedure already reported in (Marulier et al., 2012) (Marulier et al., 2015). These pulp fibres were chosen to limit the complexity of the fibrous microstructures of the papers. Some of them were red dyed to estimate the fibre orientation distribution function of the processed papers. Paper handsheets with planar random or orientated fibrous microstructures were prepared using a Rapid Köthen former (Karl Frank GMHB, Weinheim, Germany) and a dynamic handsheet former (Techpap, Grenoble, France), respectively. Then, the wet handsheets were pressed and consolidated between blotters for $3 \mathrm{~min}$ at a prescribed wet pressing pressure before being dried in the dryer of the Rapid Köthen machine system for 6 min at $85^{\circ} \mathrm{C}$. Hence, the as-processed papers did not exhibit any pronounced in-plane shrinkage nor out-of-plane deformation. To obtain papers with different volume fraction of fibres $\phi$, the wet pressing pressure was varied from 0.1 to $1.3 \mathrm{MPa}$. The thickness and the basis weight $G$ of each handsheet were measured after consistent and controlled storage (temperature $=23^{\circ} \mathrm{C}$ and relative humidity $=50 \% \mathrm{rh}$ ) to estimate the paper density $\rho_{p}$ and their volume fraction of fibres $\phi$ (Table 1$)$.

Table 1. Density $\rho_{p}$ and volume fraction of fibres $\phi$ of the fabricated low-density papers ( $\phi$ was estimated from $\rho_{p}$ and from the density of pure cellulose $\rho_{c}=1500 \mathrm{~kg} \mathrm{~m}^{-3}$ with $\phi \approx$ $\rho_{p} / \rho_{c}$ (Salmen and Fellers 1989)).

\begin{tabular}{|c|c|c|}
\hline & $\rho_{p}\left(\mathrm{~kg} \mathrm{~m}^{-3}\right)$ & $\phi$ \\
\hline \multirow{8}{*}{$\begin{array}{l}\text { papers with planar random } \\
\text { fibre orientation }\end{array}$} & 294 & 0.20 \\
\hline & 356 & 0.24 \\
\hline & 411 & 0.27 \\
\hline & 485 & 0.32 \\
\hline & 513 & 0.34 \\
\hline & 520 & 0.34 \\
\hline & 531 & 0.35 \\
\hline & 700 & 0.45 \\
\hline \multirow{12}{*}{$\begin{array}{l}\text { papers with in-plane } \\
\text { orientated fibres }\end{array}$} & 100 & 0.05 \\
\hline & 150 & 0.1 \\
\hline & 232 & 0.16 \\
\hline & 296 & 0.20 \\
\hline & 327 & 0.22 \\
\hline & 348 & 0.23 \\
\hline & 404 & 0.27 \\
\hline & 465 & 0.31 \\
\hline & 491 & 0.33 \\
\hline & 495 & 0.33 \\
\hline & 519 & 0.35 \\
\hline & 537 & 0.36 \\
\hline
\end{tabular}




\subsection{Microstructure characterisation}

To characterise the in-plane orientation distributions of fibres of the model papers, we identified more than 1200 red dyed fibres at the surface of the model papers and determined the in-plane orientation angle $\psi$ for each segment of all scanned fibres (Marulier et al., 2015). In addition, the $3 \mathrm{D}$ microstructures of the model papers were characterised using synchrotron X-ray microtomography (ID 19 beamline, ESRF, France, X-ray energy = $17.6 \mathrm{keV}$, scans with 1500 radiographs, duration $<5 \mathrm{~min}$, voxel size $=0.7 \mu \mathrm{m}^{3}$, Paganin imaging mode used for the reconstruction, field of view $1400 \times 1400 \times 500 \mu \mathrm{m}^{3}$, one 3D image per studied sample). From the $3 \mathrm{D}$ images, we extracted some fibres and fibre bonds from the fibrous networks to analyse qualitatively their morphology. In addition, several microstructure descriptors of fibres and fibre bonds were measured together with their mean values (hereafter specified with a bar). Some of them are depicted in the scheme shown in Fig. 1. Among them are the length $l$, the orientation and the curvature of centrelines of fibres, the thickness $t$ and the width $w$ of their cross sections (and the related fibre aspect ratios $r_{1}=l / w, r_{2}=w / t$ and $r_{3}=r_{1} r_{2}=l / t$ ). We also measured the number of fibre bonds per fibre $z$, the typical width $w_{b}$ and the surface $S_{b}$ of bonds as well as the bonding degree ratio $r_{b}=w_{b} / w$ (see Fig.1). These descriptors are of particular interest in the following sections. Also, it is worth noting that the bond surfaces were only estimated geometrically from the 3D images: no morphological information below the voxel size could be obtained $\left(0.7 \mathrm{\mu m}^{3}\right)$, nor physico-chemical information at the macromolecular scale.

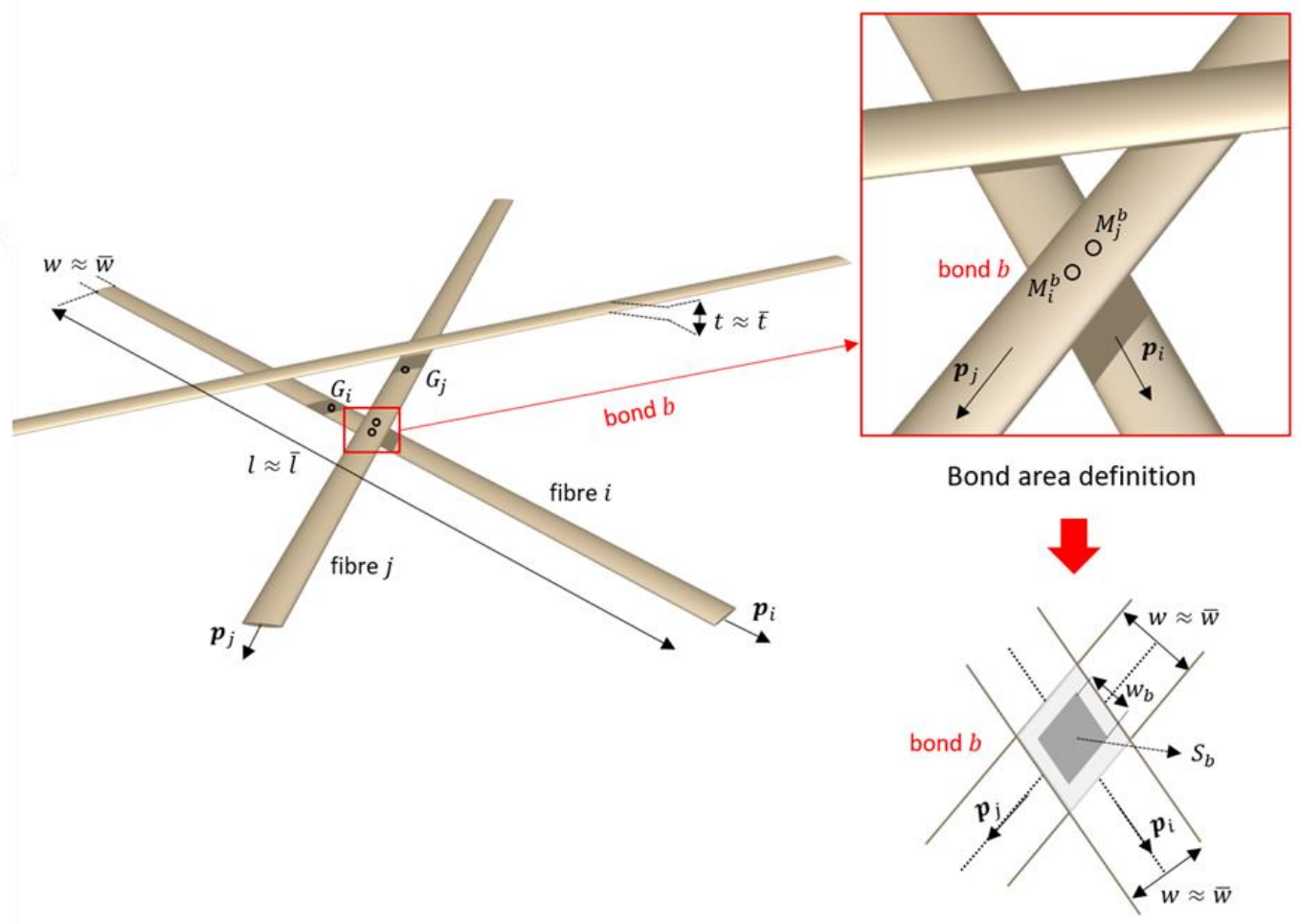

Figure 1. Idealised representation of interacting and straight fibres $i$ and $\boldsymbol{j}$ (of orientation vector $\boldsymbol{p}_{i}$ and $\boldsymbol{p}_{j}$, length $\bar{l}$, width $\overline{\boldsymbol{w}}$, thickness $\bar{t}$ ) and their fibre bonds $b$. The idealised bonding surface $S_{b}$ with its typical width $\bar{w}_{b}$ is sketched in grey in the bottom right scheme. 


\subsection{Mechanical characterisation}

Uniaxial tensile tests were performed on slender rectangular specimens (mean width $=10 \mathrm{~mm}$ and mean initial gauge length $=150 \mathrm{~mm}$ ) at a constant initial strain-rate $\dot{\varepsilon}=0.001 \mathrm{~s}^{-1}$ using a commercial horizontal tensile testing machine (Lorentzen and Wettre No. 162, tensile tester). For the in-plane orientated papers, tensile tests were performed along the longitudinal and transverse directions, i.e., in the two in-plane principal orientation directions of papers. Before testing, paper samples were stored for at least one day under controlled conditions: $T=23^{\circ} \mathrm{C}$ and $50 \% \mathrm{rh}$. The Young's moduli of the fabricated low-density papers were then estimated for each specimen following the procedure reported in (Niskanen and Krenlampi 1998). The Young's moduli of in-plane random and orientated papers were denoted $E, E_{L}$ and $E_{T}$, respectively.

\section{3 - Experimental results and discussion}

\section{1 - Microstructures of papers and modelling their fibrous connectivity}

The 3D images shown in Figs. 2-3 give typical features of the studied papers, fibres and fibre bonds. From these images several important remarks can be mentioned:

Fibre networks - Fig. 1a shows that the paper sheets consisted in thin (thickness ranging from 60 to $200 \mu \mathrm{m}$ ) and more or less densely connected fibrous networks with mostly planar fibre orientation in the midplane $\left(\mathbf{e}_{1}, \mathbf{e}_{2}\right)$ of the sheets. The mean value of the out of plane angle of the fibre centrelines was $0^{\circ}$ and the related standard deviation did not exceed $2.5^{\circ}$. As shown in Fig. 3a, the in-plane fibre orientation distribution is flat for the sheets that were fabricated with the Rapid Köthen former, revealing a planar random fibre orientation (see the corresponding second order fibre orientation tensor A (Advani and Tucker, 1987) given in Fig. 3c). Such a distribution exhibits a Gaussian-like shape for sheets processed with the dynamic handsheet former (Fig. 3b), with a preferred fibre orientation along the machine or longitudinal direction.

Fibres - Figs. 2a-b reveal that fibres were rather straight so that each fibre $i$ could be ascribed a mean tangent unit vector $\mathbf{p}_{i}$ to characterise its orientation. In addition, fibres exhibited a ribbon-like shape and cross sections with collapsed lumens. The fibres had a mean length $\bar{l} \approx 2000 \mu \mathrm{m}$, a mean width $\bar{w} \approx 30 \mu \mathrm{m}$ and a mean thickness $\bar{t} \approx 6 \mu \mathrm{m}$, so that $\bar{r}_{1} \approx 67$, $\bar{r}_{2} \approx 5$ and $\bar{r}_{3} \approx 335$. These parameters were practically constant regardless of the papermaking conditions. In addition, fibres were weakly twisted (Fig. 2): the major direction of their cross sections (of mean dimension $w$ ) was nearly parallel to the midplane $\left(\mathbf{e}_{1}, \mathbf{e}_{2}\right)$ of the sheets. Lastly, thanks to the high spatial resolution of the $3 \mathrm{D}$ images, it is possible to distinguish the presence of kinks distributed all along the fibre length (Fig. 2b): their origin could be related to the fibre extraction process and/or to fibre/cell wall growth (Page et al., 1962) (Nanko and Ohsawa 1989) (Nyholm et al., 2001) (Eder et al., 2008). 
Fibre bonds - Figs. 4a-d are 3D views of typical fibre bonds that were isolated from various 3D images of fibrous networks, as shown in Fig. 2a. These images reveal that papers exhibited various bond morphologies with practically full (Figs. 4a-b) or only partial (Figs. 4c-d) fibrefibre contact areas. We characterised these contact areas from the acquired 3D images using the mean bonding degree ratio $\bar{r}_{b}$, i.e., the mean of the ratios between the width of the bond $w_{b}$ to the considered fibre width $w$ (see Fig. 1, Marulier et al., 2015). These measurements that were extracted from a close examination of the 3D images with more than 80 measurements per images show that $\bar{r}_{b}$ was noticeably low (between 30 and 40\%) and an increasing function of the fibre content $\phi$ (Fig. 4e):

$$
\bar{r}_{b}=\alpha\left(\phi-\phi_{0}\right)
$$

with $\alpha=1.2$ and $\phi_{0}=0.02$. By considering reasonably that the bonding area $S_{b}$ and the projected contact zone $S$ are linked as $S_{b}=r_{b}^{2} S$ (see Fig. 1 and subsection 4.1), our results show that the measured bonding surface ratio $r_{b}^{2}$ should thus range between 0.01 and 0.16 . These values are much lower than the value of 0.6 measured by Kappel et al. (2009) on isolated fibre bonds extracted from a paper the wet pressing pressure of which is unknown, but probably higher than that used in this study. In addition, due to both the pronounced slenderness of fibres $\left(\bar{r}_{1}\right.$ and $\left.\bar{r}_{3} \gg 10\right)$ and the investigated fibre contents, the studied low-density papers formed connected fibrous networks. The results plotted in Fig. 4(f-g) show that the mean coordination number $\bar{z}$ ranged between 40 and 110. Thus, despite their low densities, these high values are likely to show that the studied papers can be considered as fully connected fibrous networks with a typical length between two adjacent bonds on a fibre of the same order of magnitude as the mean fibre width $\bar{w}$, i.e., as networks far from the percolation threshold (Åström et al., 2000, Alava and Niskanen 2006). In addition, the studied papers can also be considered to be far the concentration threshold where at least one internal mechanisms could arise during loading (i.e., with at least one hypostatic fibre). This will be confirmed later with our simulations, see section 5. Combined with the experimental trend given by Eq. (1), this observation tends to prove that bond micro-mechanics is expected to be a central mechanism in the elasticity of these low-density papers which exhibit numerous bonds with small bond surfaces.

From the 3D images, we showed that the mean coordination number $\bar{z}$, i.e., the mean number of fibre bonds per fibre, could be well estimated by a modified expression (Guiraud et al., 2012) of the tube model (Toll 1993), as illustrated in Figs. 4f-g:

$$
\bar{z}=4 \beta \phi\left(\frac{2}{\pi} \bar{r}_{1} \varphi_{1}+\varphi_{2}+1\right)
$$

where $\varphi_{1}$ and $\varphi_{2}$ are orientation functions that were completely determined from the knowledge of the orientation of the $N$ fibres contained in a representative volume of each studied paper (experimentally, more than 1200 measurements were made purposely): 


$$
\varphi_{1}=\frac{1}{N^{2}} \sum_{k=1}^{N} \sum_{l=1}^{N}\left\|\mathbf{p}_{k} \otimes \mathbf{p}_{l}\right\| \quad \text { and } \quad \varphi_{2}=\frac{1}{N^{2}} \sum_{k=1}^{N} \sum_{l=1}^{N}\left|\mathbf{p}_{k} \cdot \mathbf{p}_{l}\right|
$$

These orientation functions arise from geometrical arguments when considering the intersection of the fibres having an identical orientation vector $\mathbf{p}$ with a cylindrical volume surrounding a test fibre with an orientation $\mathbf{p}^{\prime}$ (Toll 1993). The first is deduced from the volume of the fibres $\mathbf{p}$ which intersect the lateral side of the cylinder, whereas the second one comes from the volume of fibres $\mathbf{p}$ which intersect the extremities of the cylinder. They are equal to 0 and $1,2 / \pi$ and $2 / \pi, \pi / 4$ and $1 / 2$, for unidirectional, planar random and $3 \mathrm{D}$ random fibre orientation, respectively. In addition, the parameter $\beta \approx 2$ in Eq. (3) was introduced by (Marulier et al., $2015)$ to account for the slightly twisted geometry of the studied fibres (Figs. 2-3).

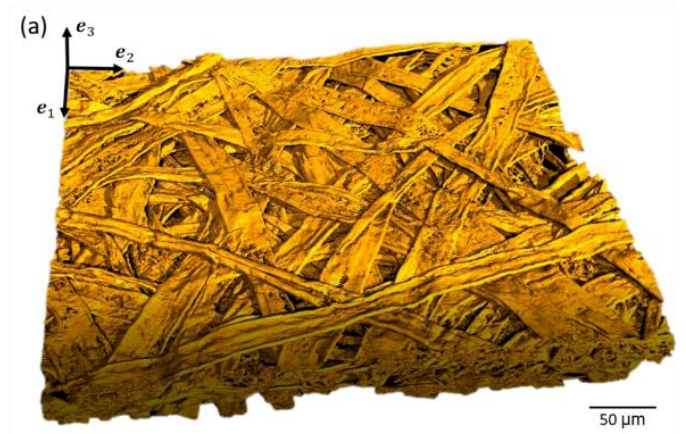

(b)

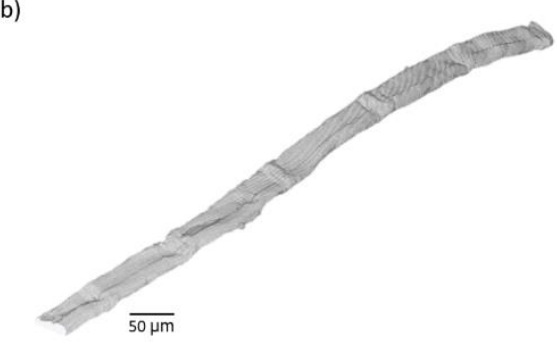

Figure 2. 3D images of (a) a fabricated low-density paper with planar random fibre orientation and a fibre content $\phi=0.323$ and (b) a single fibre that was isolated from the rest of the fibrous network. 
(a)
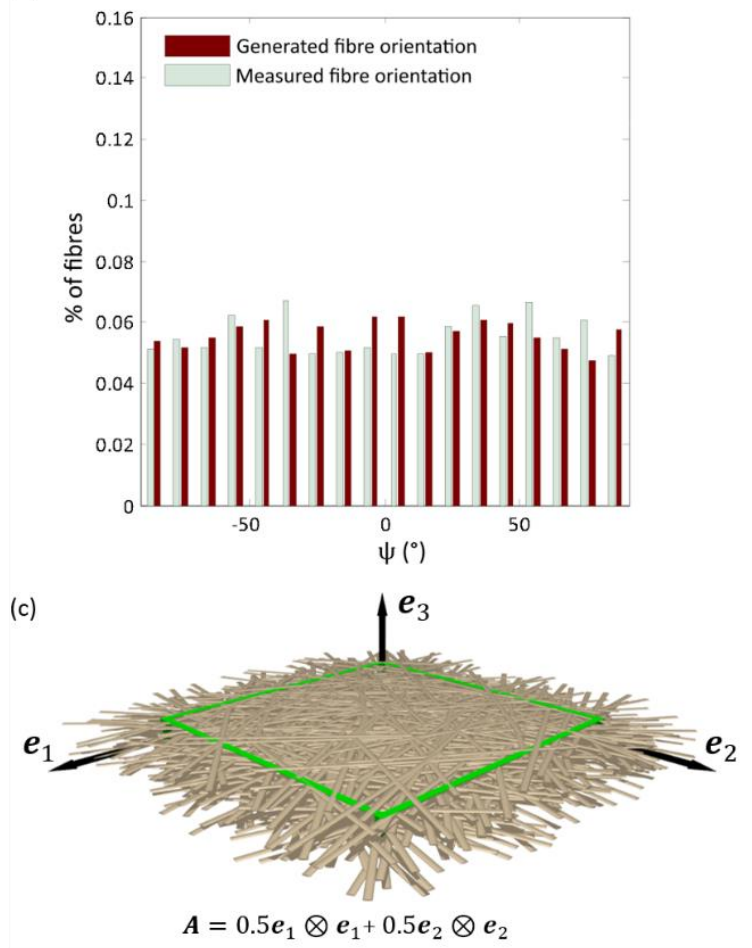

(b)

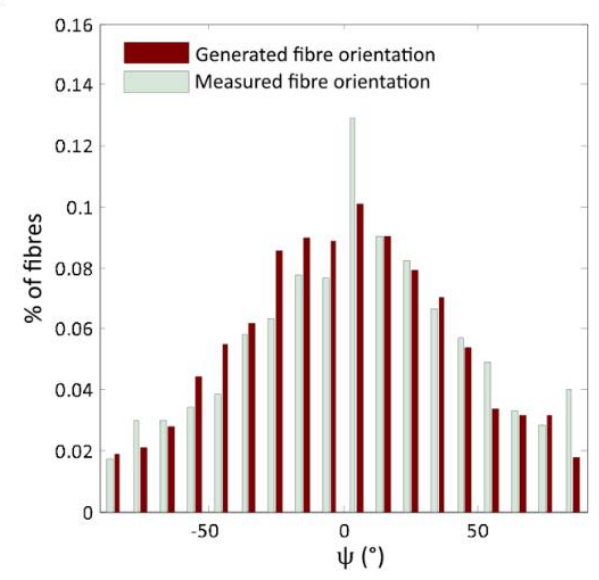

(d)

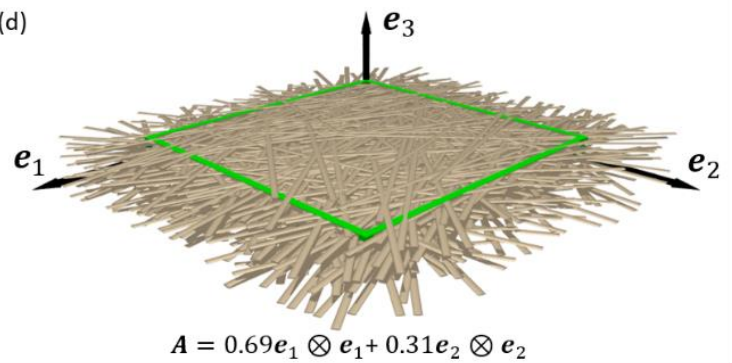

Figure 3. (a-b) Comparison of the in-plane fibre orientation distributions measured from the fabricated low-density papers with those obtained using the numerical generation process. (c-d) Idealised REVs of fibrous networks $(\phi=0.15)$ with a planar random fibre orientation (a) and a preferred fibre alignment along the $\boldsymbol{e}_{1}$-direction (b).

The evolutions of the Young's moduli $E, E_{L}$ and $E_{T}$ as a function of the fibre content $\phi$ are plotted in Fig. 5a (no relevant data was obtained for the orientated papers with the lowest density, i.e., for $\phi=0.05$, due the weakness of the measured tensile forces and the capacity of the load cell of the testing machine). Significant orthotropy is highlighted in the figure for the orientated papers with $E_{L} \gg E_{T}$. In addition, it is worth noticing from the graph depicted in Fig. 5b that the normalised elastic moduli $E / E_{\phi=0.35}, E_{L} / E_{L_{\phi=0.35}}$ and $E_{T} / E_{T \phi=0.35}$ followed the same master curve which obviously emphasises a power-law increase of the paper elasticity with $\phi$ :

$$
E / E_{\phi=0.35} \approx E_{L} / E_{L_{\phi=0.35}} \approx E_{T} / E_{T \phi=0.35} \propto \phi^{4.3}
$$

The non-linearity is principally noticed for low fibre contents, i.e., when $0.1 \leq \phi \leq 0.35$ and seems in-line with that observed earlier (Rigdahl et al., 1983, Niskanen 2011, Park et al., 2020). For example, by analysing the experimental data of Rigdahl et al. (1983), a power-law exponent of 3.6 was found. These trends are not commonly observed for standard papers with higher densities (Niskanen 2011), for which the Young's moduli follow more or less linear evolutions with the fibre contents. It tends to prove that the micromechanics of low-density papers may differ from that of denser papers. 
(a)

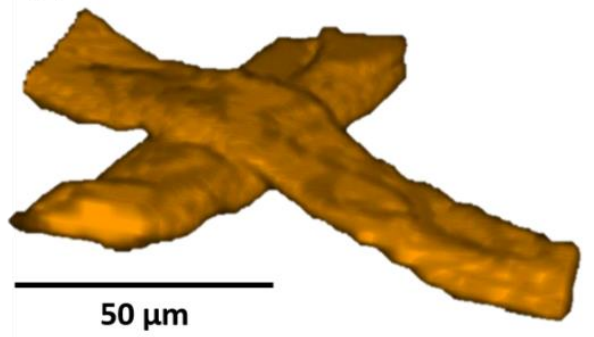

(c)

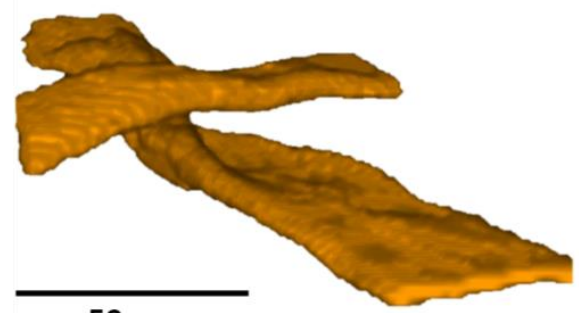

$50 \mu \mathrm{m}$ (b)

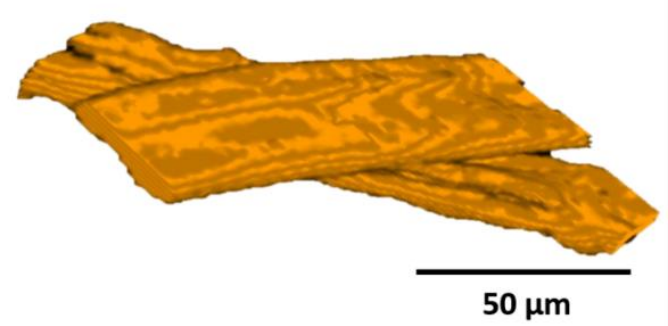

(d)

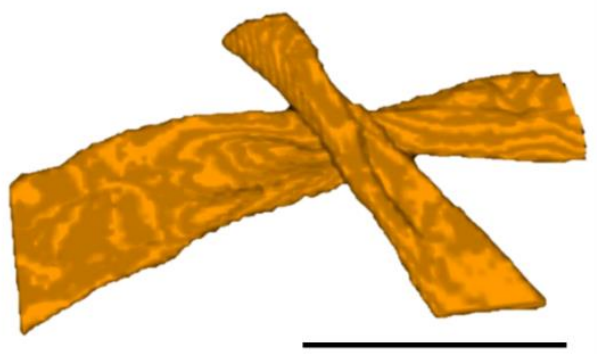

$50 \mu \mathrm{m}$

(e)

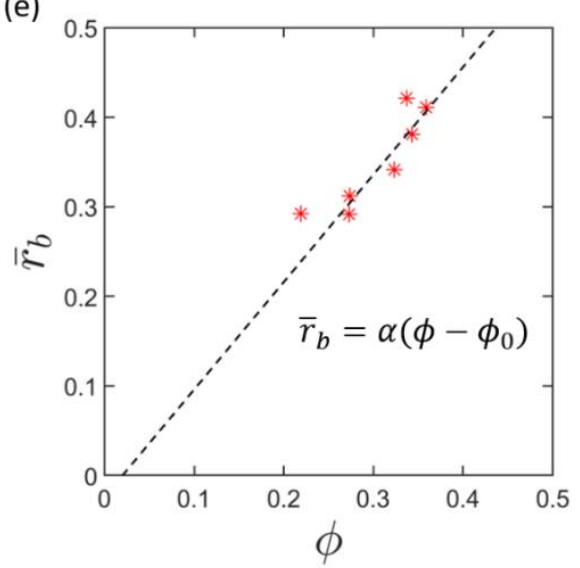

(f)

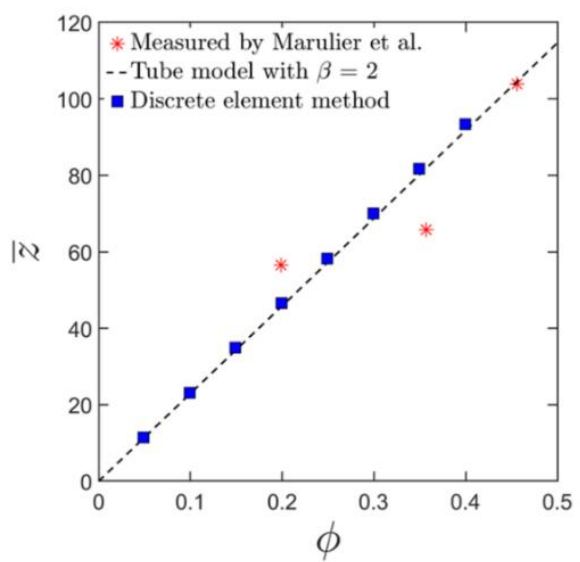

(g)

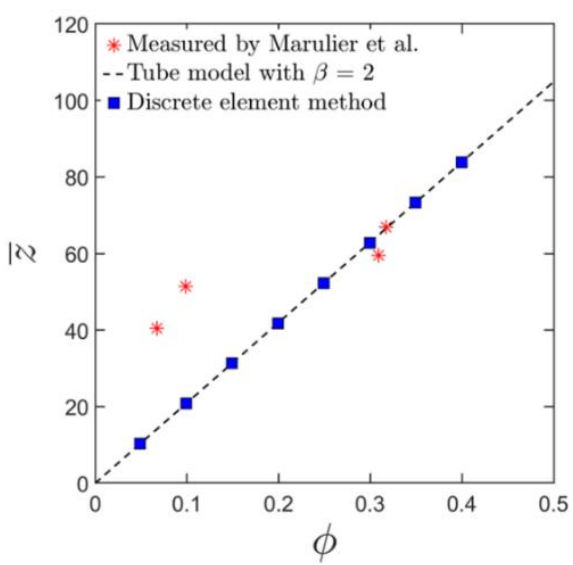

Figure 4. (a-d) Examples of $3 D$ images (voxel size $0 . r^{n} \mu m^{3}$ ) of typical fibre-fibre bonds with high (a-b) and weak ( $c$-d) contact areas. (e) Evolution of the mean bonding degree ratio $\overline{r_{b}}$ as a function of the fibre content $\phi$ for both types of model papers. The continuous line is the linear fit of Eq. (1) used in the numerical and analytical models. ( $f$-g) Mean coordination number $\bar{z}$ with $\phi$ for planar random ( $f$ ) and orientated papers (g), estimated with either 3D images (Marulier et al., 2015), or the discrete element code, or the tube model Eq (2). 


\section{2 - Elastic properties of produced papers and relevance of literature models}

The experimental evolution of $E$ were compared with the predictions proposed by some analytical models developed for fibrous materials: the models of Cox (Cox 1952), Page and Seth (Page and Seth 1980) and Wu and Dzenis (Wu and Dzenis 2005) (Fig. 5c). These approaches were chosen because they rely on very different micromechanical assumptions (see appendix 1 for their analytical expressions of $E$ ):

- Cox's model assumes that papers can be regarded as trusses of elastic straight fibres uniquely loaded in tension, without interaction with their neighbouring fibres (Cox 1952). In this approach, $E$ depends on the Young's modulus of fibres $E_{f}$ and is a linear function of the fibre content $\phi(\mathrm{Eq} .(\mathrm{A} 1))$.

- The model of Page and Seth is an extension of Cox's model (Page and Seth 1980), where it is additionally assumed that the mechanical stresses between fibres are transferred through the shear of bond regions. Hence, the elastic modulus $E$ of papers is a non-linear function of $\phi$ (Eq. (A2)), the geometry, the tensile $E_{f}$ and shear $G_{f}$ moduli of fibres, and of the relative bonded area (Page et al., 1962) (Sampson 2004).

- Wu and Dzenis proposed a micromechanical model for the stiffness of planar fibre networks with elastic straight fibres of finite length that can be stretched, bent and sheared and that are connected by rigid fibre bonds (Wu and Dzenis 2005). The resulting Young's modulus $E$ is a non-linear function of $\phi$ that depends on the fibre geometry $\left(\bar{r}_{1}, \bar{r}_{2}\right)$, the Young's modulus $E_{f}$ and the Poisson's ratio $v_{f}$ of fibres, and on the connectivity of the fibrous networks, which is effective above a critical number of fibres per unit of volume $n_{c}$ (Eq. A(3)).

To compare the prediction of these models, we used a fibre Young's modulus $E_{f}=30 \mathrm{GPa}$, which is a good estimate for softwood pines (Neagu et al., 2006) (Mansour et al., 2009). By checking that their influence was weak on the model prediction $G_{f}$ and $v_{f}$ were set to $3 \mathrm{GPa}$ and 0.3. Lastly, the critical number of fibres per unit of volume $n_{c}$ was set to 5.7 , following $2 \mathrm{D}$ estimates of the literature (Åström et al., 2000) (Alava and Niskanen 2006). Fig. 5c shows that the aforementioned models do not provide relevant prediction for the elastic modulus $E$ of the studied papers. None of them is able to capture the non-linear power-law evolution of $E$ with $\phi$ observed at low fibre contents. The models proposed by Cox and Page and Seth are linear and nearly affine functions of the fibre content $\phi$, respectively (even if a non-linear evolution for the RBA with $\phi$ is taken into account in the second model). It is interesting to note that the model of $\mathrm{Wu}$ and Dzenis (Wu and Dzenis 2005) predicts a non-linear evolution of $E$ with $\phi$. However, this non-linearity is not as marked as that observed experimentally so that the model diverges from the experimental data. Hence, results shown in Fig. 5c suggest that the elastic behaviour of the considered low-density papers is ruled by elementary deformation micro-mechanisms different from those often assumed in the analytical models. In particular, these models do not properly account for the contribution of the numerous cohesive and deformable fibre bonds nor for the peculiar geometry of these bonds (Fig. 4). 
(a)

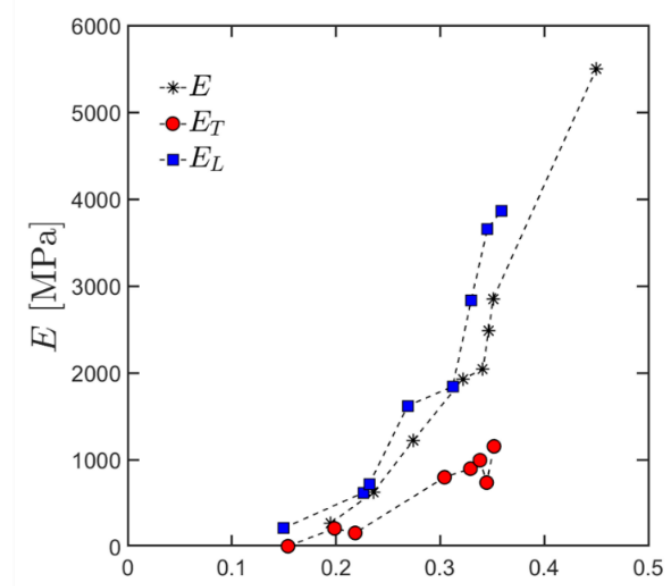

(b)
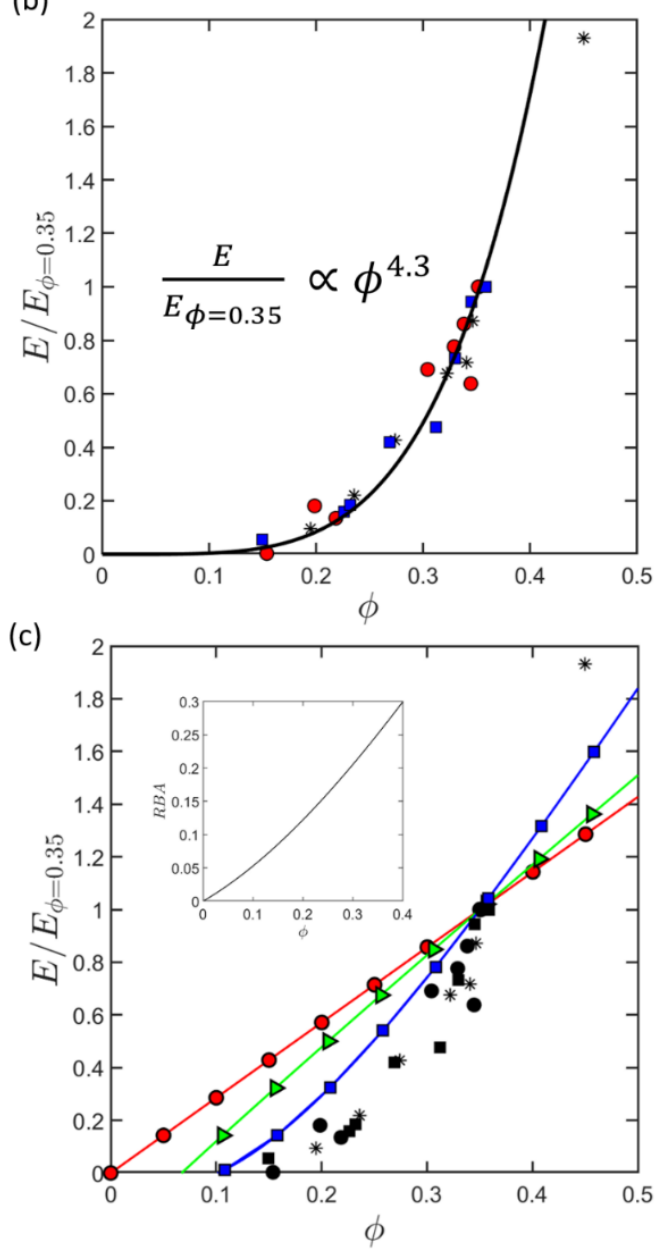

Figure 5. (a) Evolutions of the elastic moduli with the fibre content $\phi$ for planar random (black symbols) and orientated (red and blue symbols) low-density papers. (b) Normalised moduli $E / E_{\phi=0.35}, E_{T} / E_{T \phi=0.35}$ and $E_{L} / E_{L \phi=0.35}$ as a function of $\phi$ (the continuous line is the power-law Eq. (1)). (c) Evolution of the same normalised moduli with $\phi$ and comparison with the normalised predictions given by the Cox's model $\left(E_{c o x} / E_{c o x \phi=0.35}\right.$ continuous red line), the model of Page and Seth $\left(E_{P S} / E_{P S \phi=0.35}\right.$ continuous green line) and the model of $W u$ and Dzenis ( $E_{W D} / E_{W D \phi=0.35}$ continuous blue line). The inset shows the evolution of the RBA with $\phi$ (Sampson 2008) (Sampson 2004) that was used for the model of Page and Seth. 


\section{4 - A new micromechanical model focusing on fibre bonds}

In light of (i) the experimental data gathered in the previous section and (ii) the difficulty of classical analytical micromechanical models to predict the elastic properties of low-density papers, we herein propose novel 3D network models for the mechanics of paper materials, valid for the low density regime. The models were built on several assumptions related both to the fibrous microstructures and fibre scale deformation mechanisms of papers. The approach assumes that the micromechanics of bonds plays a central role. We introduced these features into an upscaling technique, namely the homogenisation method for discrete structures, in which additional kinematic assumptions were finally stated to obtain compact analytical estimates for the stiffness of paper materials.

\section{1 - Idealised microstructure}

From the analysis summarised in the previous section, we considered several assumptions. Thus, typical Representative Elementary Volumes (REVs) of the studied papers were seen as thin but 3D discrete structures with $N$ cellulosic fibres $i$ of centre of mass $G_{i}$ and mean orientation vectors $\mathbf{p}_{i}$. Fibres exhibited planar fibre orientation and were considered as straight and slender beams of length $\bar{l}$, with flat elliptical cross section of area $\pi \bar{w} \bar{t} / 4, \bar{w}$ and $\bar{t}$ being the major and minor axes of the ellipse base, respectively oriented parallel and perpendicular to the midplane $\left(\mathbf{e}_{1}, \mathbf{e}_{2}\right)$ of the paper sheets (Fig. 1). Also, we assumed that there is a good separation of scale between the size of the REVs, of the same order of magnitude than the fibre length $\bar{l}$, and the macroscopic size of the paper sheets $L$, so that the scale separation parameter $\epsilon$ is small, i.e., $\epsilon=\bar{l} / L \ll 1$. In addition, a fibre bond between two contacting fibres $i$ and $j$ is denoted $b$. On fibre $i$ (resp. $j$ ), the bond is located just in front of the centreline point $M_{i}^{b}$ $\left(\right.$ resp. $M_{j}^{b}$ ) of curvilinear abscissa $s_{i}^{b}$ (resp. $s_{j}^{b}$ ). The bond exhibits a contact area $S_{b}$ which depends on the mean fibre width $\bar{w}$, mean bonding degree ratio $\bar{r}_{b}$, and the relative orientation of fibres $i$ and $j$. As sketched in Fig. 1 in grey, and for the sake of simplicity, we considered that a good estimate of $S_{b}$ was a weighted rhomb surface of with $\bar{w}_{b}=\bar{r}_{b} \bar{w}$ defined by the projection of the contact surface of connected fibres $(i$ and $j$ ) onto their normal contact plane (Le Corre et al., 2005)(Guiraud et al., 2012)(Martoïa et al., 2016a) (Martoïa et al., 2016b):

$$
S_{b} \approx \frac{\bar{w}_{b}^{2}}{\left\|\mathbf{p}_{i} \times \mathbf{p}_{j}\right\|}=\frac{\bar{r}_{b}^{2} \bar{w}^{2}}{\left\|\mathbf{p}_{i} \times \mathbf{p}_{j}\right\|}
$$

\section{2 - Idealised micromechanics}

To upscale the elastic properties of the considered fibrous networks, we assumed that fibres were slender rigid beams linked with linear elastic bonds $b$ (Fig. 1). This apparently strong assumption is supported by the experimental observations reported in the previous section. Indeed, due to both the high connectivity of the studied papers and the low bonding degree ratio $\bar{r}_{b}$ (Fig. 4), we assumed that the fibre deformation far from the bonds could be neglected compared with the deformation mechanisms arising inside the bond interfaces and their close 
vicinity. Consequently, when the considered fibrous networks were subjected to a macroscopic in-plane infinitesimal displacement gradient $\boldsymbol{\nabla} \overline{\mathbf{u}}$, the in-plane infinitesimal displacement $\mathbf{u}_{i}^{b}$ of the fibre centreline point $M_{i}^{b}$ is:

$$
\mathbf{u}_{i}^{b}=\mathbf{u}_{i}+s_{i}^{b} \theta_{i} \mathbf{e}_{3} \times \mathbf{p}_{i}
$$

where $\mathbf{u}_{i}$ and $\theta_{i}$ are the in-plane displacement of $G_{i}$ and rotation angle of the fibre $i$ around the out-of-plane direction $\mathbf{e}_{3}=\mathbf{e}_{1} \times \mathbf{e}_{2}$, respectively. The displacements and rotations of fibres $i$ and $j$ induced the deformation of the bond $b$, which induced an in-plane shear reaction force $\mathbf{f}_{b}$ and an out-of-plane shear reaction moment noted $m_{b} \mathbf{e}_{3}$ (expressed in the centre of the bond), both being exerted by fibre $j$ on fibre $i$. We proposed simple expressions for $\mathbf{f}_{b}$ and $m_{b}$ (Le Corre et al., 2005):

$$
\begin{gathered}
\mathbf{f}_{b}=\bar{k}_{b} S_{b} \Delta \mathbf{u}_{b}, \\
m_{b}=\bar{k}_{b} \frac{\pi}{4} S_{b}^{2} \Delta \theta_{b},
\end{gathered}
$$

where $\bar{k}_{b}$ is the mean specific bond stiffness (the bond interface and its vicinity), and where $\Delta \mathbf{u}_{b}$ and $\Delta \theta_{b}$ are the relative displacement and rotation between the connected fibres $i$ and $j$ :

$$
\begin{gathered}
\Delta \mathbf{u}_{b}=\mathbf{u}_{j}-\mathbf{u}_{i}+\mathbf{e}_{3} \times\left(s_{j}^{b} \theta_{j} \mathbf{p}_{j}-s_{i}^{b} \theta_{i} \mathbf{p}_{i}\right), \\
\Delta \theta_{b}=\theta_{j}-\theta_{i} .
\end{gathered}
$$

Hence, neglecting the external volume forces and moments, and introducing $\mathcal{B}_{i}$ the set of bonds of fibre $i$, the momentum balance equations that govern the quasi-static motion of the set $\mathcal{N}$ of the $N$ fibres $i$ contained in a REV is the following two-dimensional mechanical problem (Le Corre et al., 2004) (Le Corre et al., 2005) (Martoïa et al., 2016a):

$$
\forall i \in \mathcal{N},\left\{\begin{array}{c}
\sum_{\mathcal{B}_{i}} \mathbf{f}_{b}=\mathbf{0} \\
\sum_{\mathcal{B}_{i}} m_{b} \mathbf{e}_{3}=\sum_{\mathcal{B}_{i}} s_{i}^{b} \mathbf{f}_{b} \times \mathbf{p}_{i}
\end{array}\right.
$$

\section{4 - Theoretical upscaling}

It is interesting to note the strong analogy between the micromechanical problem presented in the previous subsection and the one developed by Le Corre et al. to model the rheology of highly concentrated fibre suspensions with planar fibre orientation and Newtonian fibre-fibre interactions (Le Corre et al., 2004) (Le Corre et al., 2005). Indeed, the elastic bond interaction forces and moments, the infinitesimal displacement and rotation fields of the fibres that are considered here, could be, respectively, replaced by the Newtonian viscous interaction forces and moments, the translational and rotational velocity fields of the fibres in the suspensions that were studied by Le Corre et al. Hence, using this analogy together with the theoretical 
developments carried out in (Le Corre et al., 2004) with the discrete adaptation (Tollenaere and Caillerie 1998) of the homogenisation method for periodic structures with multiscale asymptotic expansions (Bensoussan et al. 1978) (Sanchez-Palencia 1980), several interesting results can be mentioned. Among them, it is possible to show that the local reaction forces $\mathbf{f}_{b}$ and moments $m_{b}$ keep their generic form Eqs. (7)-(8) with the following relevant approximations:

$$
\begin{gathered}
\Delta \mathbf{u}_{b} \approx \delta \mathbf{u}_{j}-\delta \mathbf{u}_{i}+\nabla \overline{\mathbf{u}} \cdot \boldsymbol{\xi}_{b}+\mathbf{e}_{3} \times\left(s_{j}^{b} \bar{\theta}_{j} \mathbf{p}_{j}-s_{i}^{b} \bar{\theta}_{i} \mathbf{p}_{i}\right), \\
\Delta \theta_{b} \approx \bar{\theta}_{j}-\bar{\theta}_{i} .
\end{gathered}
$$

where $\delta \mathbf{u}_{i}$ (and $\delta \mathbf{u}_{j}$ ) are the first order displacement fluctuations around the macroscopic displacement field $\overline{\mathbf{u}}, \bar{\theta}_{i}$ (and $\bar{\theta}_{j}$ ) the first order rotations of fibres and where:

$$
\boldsymbol{\xi}_{b}=\mathbf{G}_{i} \mathbf{G}_{j}=s_{i}^{b} \mathbf{p}_{i}-s_{j}^{b} \mathbf{p}_{j} .
$$

In addition, if the separation of scale is good such as here $(\epsilon \ll 1)$, it can be proved that the equivalent macroscale continuum is a Cauchy medium with a macroscale symmetric stress tensor $\boldsymbol{\sigma}$ that takes the following generic discrete form:

$$
\boldsymbol{\sigma}=\frac{1}{V_{\text {rev }}} \sum_{\mathcal{B}} \boldsymbol{\xi}_{b} \otimes \mathbf{f}_{b},
$$

where $V_{\text {rev }}$ is the volume of the REV and $\mathcal{B}$ the set of the $B$ bonds contained in it. By introducing $B / V_{\text {rev }}=n \bar{z} / 2$, the number of bonds per unit volume and $n$ the number of fibres per unit volume $\left(n=N / V_{\text {rev }}\right)$, and thanks to Eq. (5), $\boldsymbol{\sigma}$ can be expressed as follows:

$$
\boldsymbol{\sigma}=\bar{k}_{b} \bar{r}_{b}^{2} \bar{w}^{2} \frac{n \bar{z}}{2} \frac{1}{B} \sum_{\mathcal{B}} \frac{1}{\left\|\mathbf{p}_{i} \times \mathbf{p}_{j}\right\|} \xi_{b} \otimes \Delta \mathbf{u}_{b} .
$$

Finally, the homogenisation method allows proving rigorously that $\boldsymbol{\sigma}$ is a linear function of the macroscale strain tensor $\boldsymbol{\varepsilon}=\left(\boldsymbol{\nabla} \overline{\mathbf{u}}+\boldsymbol{\nabla} \overline{\mathbf{u}}^{t}\right) / 2$ (Le Corre et al., 2004):

$$
\sigma=\mathbb{C}: \varepsilon
$$

where $\mathbb{C}$ is the symmetric macroscopic elastic stiffness tensor related to fibre-fibre bonds.

\section{5 - Estimates of the elastic stiffness tensors}

In order to estimate the stiffness tensor $\mathbb{C}$, we used two strategies, i.e., a numerical one based on discrete element simulation and an analytical one with additional assumptions on the fibre kinematics.

\subsection{1 - Discrete element simulation}


Taking advantage of the aforementioned analogy, we used the numerical method proposed by Le Corre et al. (Le Corre et al., 2005) (Dumont et al., 2009). The components of the stiffness tensors $\mathbb{C}$ were thus calculated by (i) generating realistic REVs, (ii) subjecting them to three independent macroscale strains $\boldsymbol{\varepsilon}$, e.g., $\boldsymbol{\varepsilon}^{I}=\mathbf{e}_{1} \otimes \mathbf{e}_{1}, \boldsymbol{\varepsilon}^{I I}=\mathbf{e}_{2} \otimes \mathbf{e}_{2}, \boldsymbol{\varepsilon}^{I I I}=\left(\mathbf{e}_{1} \otimes \mathbf{e}_{2}+\mathbf{e}_{2} \otimes\right.$ $\left.\mathbf{e}_{1}\right) / 2$ and solving the linear system Eqs. (7)-(8),(11)-(13) to estimate the kinematic fields $\delta \mathbf{u}_{i}$ and $\bar{\theta}_{i}$ related to these elementary macroscopic strain fields, (iii) computing the resulting macroscopic Cauchy stress tensors $\boldsymbol{\sigma}$ with Eq. (16), i.e., $\boldsymbol{\sigma}^{I}, \boldsymbol{\sigma}^{I I}, \boldsymbol{\sigma}^{I I I}$, respectively, and (iv) identifying the components of $\mathbb{C}$ with Eq. (17). For step (ii), we used the discrete element solver developed in (Le Corre et al., 2005). For step (i), to generate numerical REV's that mimicked the fibrous microstructures of the fabricated papers, we used a simple deterministic technique inspired from the statistical tube model Eq. (2). Briefly, $N$ straight fibres $i$ of lenght $\bar{l}$ with elliptical cross section of area $\pi \bar{w} \bar{t} / 4$ and with orientation vectors $\mathbf{p}_{i}$ parallel to the paper sheet midplane $\left(\mathbf{e}_{1}, \mathbf{e}_{2}\right)$ were generated inside REVs of volume $V_{\text {rev }}=l_{\text {rev }}^{2} t_{\text {rev }}$. The in-plane dimension $l_{\text {rev }}$ of the REVs was set larger than the fibre length $\bar{l}$ to avoid generating continuous fibres. Practically, the values of $l_{\text {rev }}$ and $h_{\text {rev }}$ were set to $1.5 \bar{l}$ and $10 \bar{t}$, respectively, and we checked that above these values, results remained unchanged. Thus, fibrous networks were generated with various fibre contents $\phi=n \pi \bar{w} \bar{t} \bar{l} / 4$ ranging from 0.05 to 0.5 , with various Gaussian-based in-plane fibre orientation distributions, from planar random to highly orientated along the $\mathbf{e}_{1}$ direction. Figs. 3c-d show two examples of typical idealised fibrous networks with random or preferred fibre orientations that were generated using this procedure. Figs. 3a-b also show that the generation procedure allowed obtaining fibrous networks with orientation distributions close to those measured experimentally. The connectivity of the generated fibrous networks was assessed using a deterministic soft-core approach: a rectangular control volume size $2 \beta \bar{l} \bar{w} \bar{t}$ centered on the centerline of each fibre $i$ was defined, each fibre the centreline of which intersected the control volume was added to the connectivity sets $\mathcal{B}_{i}$ and $\mathcal{B}$. As shown in Figs. 4f-g, the computed mean coordination number $\bar{z}$ followed the same evolution than that predicted by the modified statistical tube model Eq. (2), i.e., close to the experimental data deduced from the 3D images. Lastly, to run the simulation, it is worth noting that the bonds of the REVs were ascribed a mean bonding degree ratio $\bar{r}_{b}$ which followed the affine function Eq. (1). For a given couple of fibre orientation and content, simulations were repeated 10 times to better estimate trends.

\subsection{2 - Analytical estimates}

To build compact analytical expressions for the elastic stiffness tensors of papers with relevant microstructure and micromechanical parameters, additional assumptions were stated both on the fibrous microstructures and on the kinematics of fibres. Their relevance was checked using the microstructure generator and discrete element simulations:

1. Thanks to the results plotted in Figs. 4f-g, we assumed that the modified tube model Eq (2) was a proper prediction of the mean coordination number $\bar{z}$.

2. We also supposed that the two terms inside the summation of Eq. (16) were weakly correlated so that $1 /\left\|\mathbf{p}_{i} \times \mathbf{p}_{j}\right\|$ was extracted from the summation and approximated by its mean value. This is equivalent to stating that the bond area $S_{b}$ can be approximated by its mean value $\bar{S}_{b}$ : 


$$
S_{b}=\frac{\bar{r}_{b}^{2} \bar{w}^{2}}{\left\|\mathbf{p}_{i} \times \mathbf{p}_{j}\right\|} \approx \bar{S}_{b} \approx \frac{\bar{r}_{b}^{2} \bar{w}^{2}}{\varphi_{1}}
$$

3. The infinitesimal displacement field $\mathbf{u}_{i}$ of the centre of mass $G_{i}$ was assumed to be an affine function of the macroscopic displacement gradient $\boldsymbol{\nabla} \overline{\mathbf{u}}$. This assumption led to neglecting the first right-hand term in the expression (12) of $\Delta \mathbf{u}_{b}, i . e ., \delta \mathbf{u}_{j}-\delta \mathbf{u}_{i} \approx \mathbf{0}$.

4. The rotation of the fibre $i$ denoted $\mathbf{e}_{3} \times \bar{\theta}_{i} \mathbf{p}_{i}$ was assumed to follow that subjected by the macroscale transformation:

$$
\mathbf{e}_{3} \times \bar{\theta}_{i} \mathbf{p}_{i}=\boldsymbol{\nabla} \overline{\mathbf{u}} \cdot \mathbf{p}_{i}-\mathbf{p}_{i} \otimes \mathbf{p}_{i} \otimes \mathbf{p}_{i}: \boldsymbol{\varepsilon}
$$

Thus, accounting for the two previous assumptions, $\Delta \mathbf{u}_{b}$ was rewritten:

$$
\Delta \mathbf{u}_{b}=\left(s_{i} \mathbf{p}_{i} \otimes \mathbf{p}_{i} \otimes \mathbf{p}_{i}-\mathrm{s}_{j} \mathbf{p}_{j} \otimes \mathbf{p}_{j} \otimes \mathbf{p}_{j}\right): \boldsymbol{\varepsilon},
$$

and after some analytical developments detailed in Appendix 2, a simple and compact analytical estimate of the stiffness tensor $\mathbb{C}$ was obtained:

$$
\mathbb{C}=\frac{4 \beta \bar{k}_{b} \bar{l} \phi^{2}\left(\frac{2}{\pi} \bar{r}_{1} \varphi_{1}+\varphi_{2}+1\right) \bar{r}_{b}^{2} \bar{r}_{2}}{3 \pi \varphi_{1}}\left(1-\frac{1}{4 \phi\left(\frac{2}{\pi} \bar{r}_{1} \varphi_{1}+\varphi_{2}+1\right)}\right) \mathbb{A},
$$

where $\mathbb{A}$ is the fourth-order fibre orientation tensor defined as (Advani and Tucker 1987):

$$
\mathbb{A}=\frac{1}{N} \sum_{i=1}^{N} \mathbf{p}_{i} \otimes \mathbf{p}_{i} \otimes \mathbf{p}_{i} \otimes \mathbf{p}_{i}
$$

Eq. (21) clearly emphasises the role played by the fibre geometry $\left(\bar{l}, \bar{r}_{1}, \bar{r}_{2}\right)$, content $(\phi)$ and orientation $\left(\mathbb{A}, \varphi_{1}, \varphi_{2}\right)$ as well as the key role played by the bond stiffness $\left(\bar{k}_{b}\right)$ and bonding degree ratio $\left(\bar{r}_{b}\right)$ on the macroscale stiffness $\mathbb{C}$. It is worth noticing that for slender fibres and highly connected fibrous networks such as those investigated in this study, i.e., when $\bar{r}_{1} \gg 1$ and $\bar{z} \gg 10$, Eq. (21) can be drastically simplified to:

$$
\mathbb{C}=\frac{8}{3 \pi^{2}} \beta \bar{k}_{b} \bar{l} \bar{r}_{3} \bar{r}_{b}^{2} \phi^{2} \mathbb{A}
$$

In this case, $\mathbb{C}$ should thus follow linear evolutions with the fibre length $\bar{l}$, the fibre aspect ratio $\bar{r}_{3}$, and the fibre orientation tensor $\mathbb{A}$. In addition, the effect of the bonding ratio is noticeable, quadratic, and accounting for the measured affine evolution of $\bar{r}_{b}$ with $\phi$ (Eq. (1)), $\mathbb{C}$ should thus, at least for the low-density papers studied in this work, increase as a polynomial function of degree 4 of the fibre content $\phi$. 


\section{5 - Model prediction and discussion}

\section{1 - Numerical results}

By taking advantage of the analogy of the present problem with that treated in (Le Corre et al., 2005), we have shown that the studied fibrous microstructures exhibit orthotropic elasticity and have also checked that the principal directions of anisotropy correspond to the principal orthogonal directions of the generated fibrous microstructures, i.e., $\mathbf{e}_{1}$ and $\mathbf{e}_{2}$. Hence, it was possible to extract from the three elementary macroscale strains $\boldsymbol{\varepsilon}^{I}, \boldsymbol{\varepsilon}^{I I}, \boldsymbol{\varepsilon}^{I I I}$ the REVs were subjected to (and from the computation of the corresponding macroscale stresses $\boldsymbol{\sigma}^{I}, \boldsymbol{\sigma}^{I I}, \boldsymbol{\sigma}^{I I I}$ ), the expressions of the longitudinal $E_{L}$ and transverse $E_{T}$ Young's moduli of the papers, as well as their in-plane shear modulus $G_{L T}$ and in-plane Poisson ratios $v_{L T}$ and $v_{T L}$, assuming an inplane stress state: $E_{L}=\sigma_{11}^{I}\left(1-v_{L T} v_{T L}\right), E_{T}=\sigma_{22}^{I I}\left(1-v_{L T} v_{T L}\right), G_{L T}=\sigma_{12}^{I I I}, v_{L T}=\sigma_{22}^{I} / \sigma_{11}^{I}$, $v_{T L}=\sigma_{11}^{I I} / \sigma_{22}^{I I}$. Fig. 6 shows the evolutions of $E_{L}, E_{T} G_{L T}, v_{L T}$ and $v_{T L}$ with the fibre content $\phi$ for papers with planar random fibre orientation and with a preferred fibre orientation (Fig. $3(\mathrm{a}, \mathrm{b}))$. These predictions were obtained using a specific shear bond stiffness $\bar{k}_{b}=2000 \mathrm{~N} \mathrm{~mm}^{-3}$. The relevance of the value of $\bar{k}_{b}$ will be discussed in subsection 5.3. From these figures, several remarks can be drawn:

- Even for the lowest investigated fibre content, our simulation proved that there was no internal mechanism within the studied fibrous structures, i.e., the generated REVs contained no hypostatic fibre. Instead, the mean coordination number $\bar{z}$ was numerically found to be close to 10 for the lowest concentration. This reinforces our experimental observations: the considered low-density papers are fully connected fibrous networks, far from the percolation threshold of these systems.

- For a fibre content $\phi$ greater than 0.1, Fig. 6 shows that the scattering of the numerical results (marks) around the mean fitted values (lines) was rather weak and mainly ascribed to the generation procedure: fibres had random positions in the REV's and the resulting fibre orientation distributions were not exactly the same every time.

- Papers with 2D planar random fibre orientation exhibited in-plane isotropy, i.e., $E=E_{L}=E_{T}, v=v_{L T}=v_{T L}$ and $G=G_{L T}=E_{L} / 2\left(1+v_{L T}\right)$. It is interesting to notice that the associated Poisson ratio $v$ was more or less constant and did not depend on the fibre content. For the considered $2 \mathrm{D}$ planar random fibre orientation, $v$ was close to a mean value of $\approx 0.33$. This value is similar to that reported in the literature for analytical or numerical discrete models, even if the adopted micromechanics differs from that considered in those studies (Cox 1952) (Heyden 2000) (Heyden and Gustafsson 1998) (Wu and Dzenis 2005). For example, the analytical model proposed by Cox (Cox 1952 ) revealed that fibre networks with planar random fibre orientation exhibited a Poisson ratio of $v=1 / 3$. In addition, Heyden and Gustafsson (Heyden and Gustafsson 1998) also found similar results using discrete element simulation for the prediction of the properties of $2 \mathrm{D}$ networks consisting of short fibres exhibiting finite tensile and bending elastic stiffnesses and elastic bonds.

- A pronounced orthotropy was observed for the fibre networks with a preferred fibre orientation along the $\mathbf{e}_{1}$-direction, i.e., $E_{L} \approx 3 E_{T}$ with $E_{T}<E<E_{L}$ and $v_{T L} \approx 3 v_{L T}$ with $v_{T L}<v<v_{L T}$. 
(a)

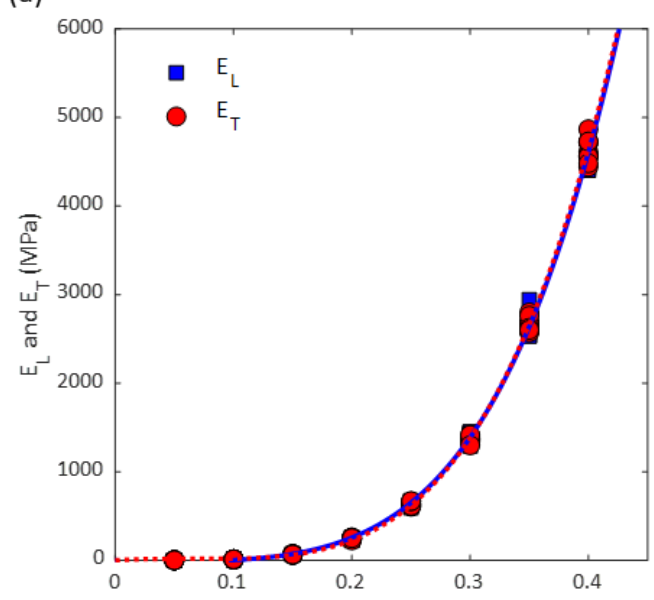

(c)

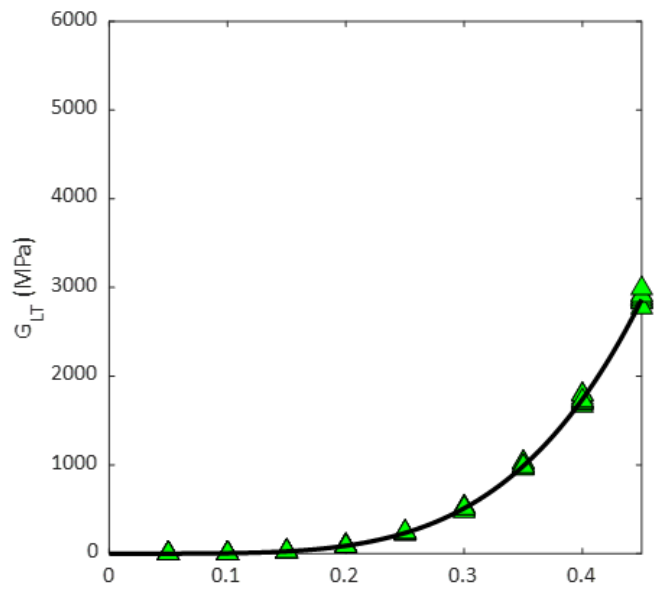

(e)

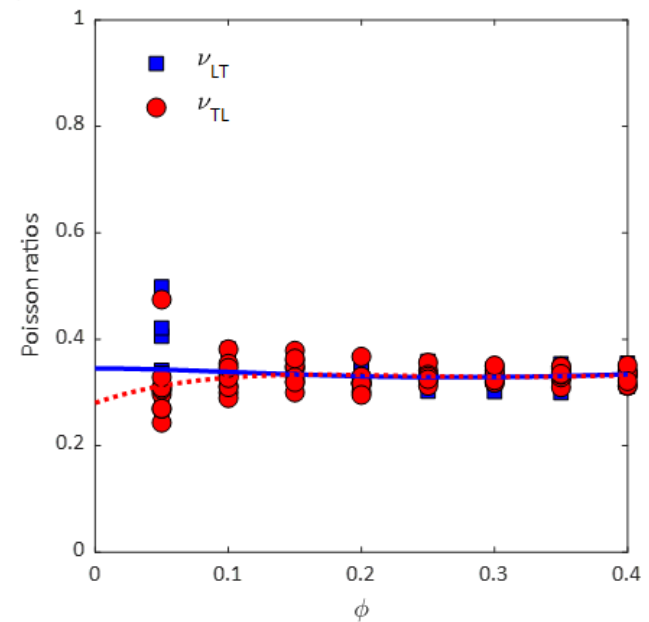

(b)

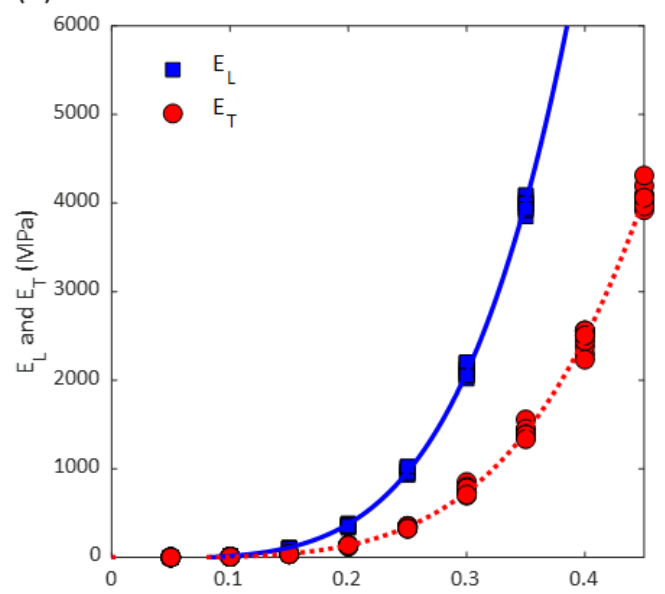

(d)

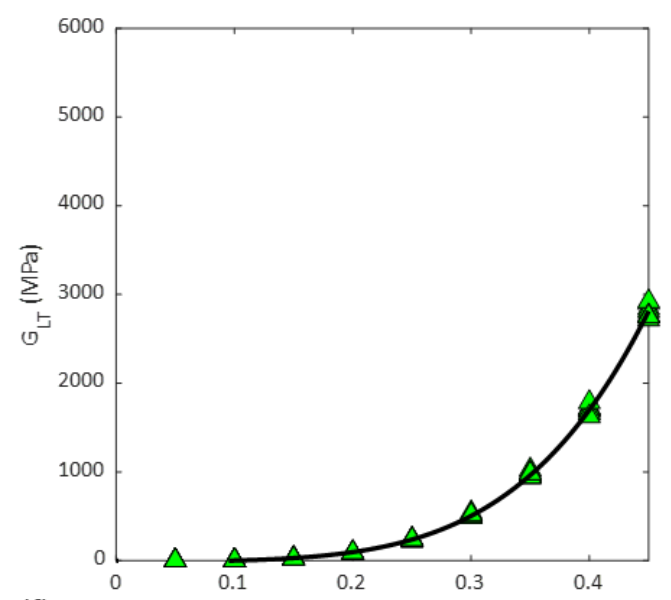

(f)

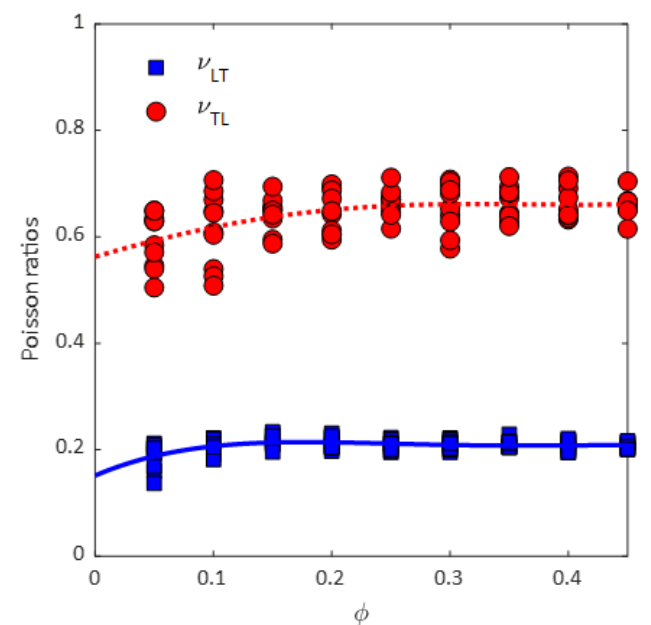

Figure 6. Evolution of $E_{L}, E_{T} G_{L T}, v_{L T}$ and $v_{T L}$ with the fibre content $\phi$ for fibrous networks with a planar random fibre orientation $(a, c, e)$ and a moderate fibre alignment along the $\boldsymbol{e}_{1}$-direction $(b, d, f)$, similar to that reported in Figs. 2c-d. The symbols correspond to numerical results and the lines to polynomial fits used to emphasise trends. 

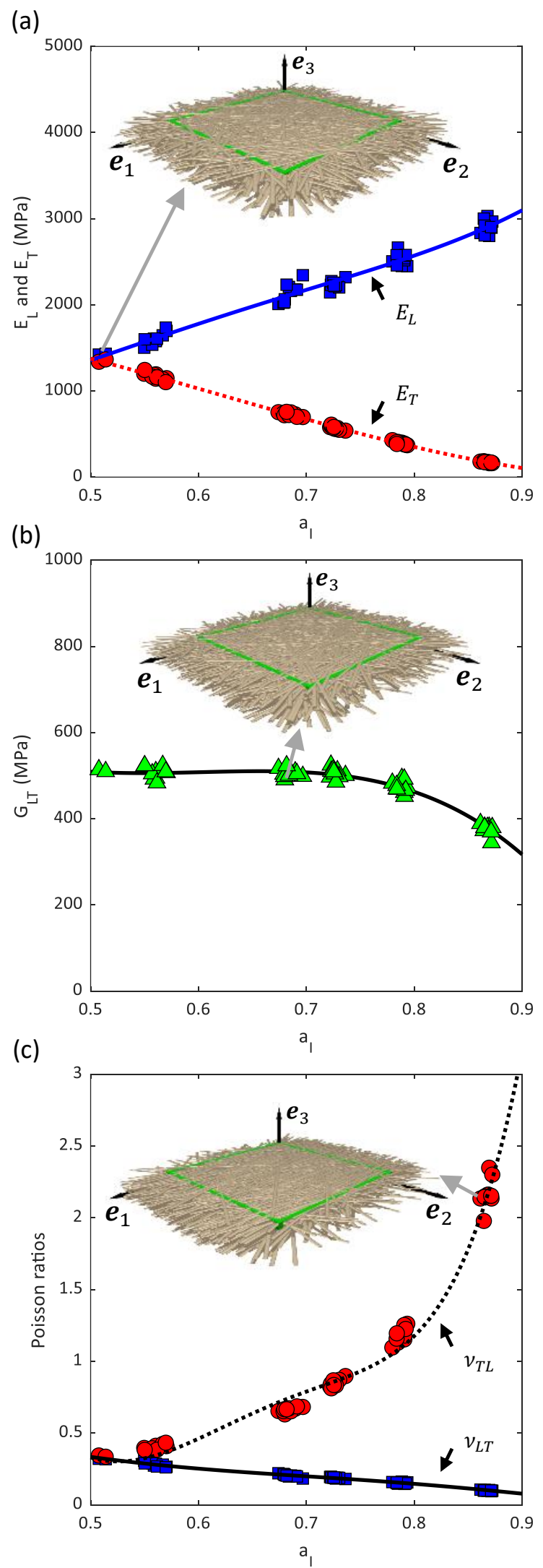

Figure 7. Evolution of the elastic parameters $E_{L}, E_{T} G_{L T}, v_{L T}$ and $v_{T L}$ with the major principal value $a_{I}$ of the second order fibre orientation tensor $\boldsymbol{A}(\phi=0.3)$. Symbols are numerical results and lines correspond to polynomial fits used to emphasise trends. 
To better highlight the effect of fibre orientation, Fig. 7 shows the evolutions of $E_{L}, E_{T} G_{L T}$, $v_{L T}$ and $v_{T L}$ (with $\phi=0.3$ ) as a function of the major principal value $a_{I}$ of the second order fibre orientation tensor $\mathbf{A}$ (for a planar random network $a_{I}=0.5$, whereas for fully aligned fibres $a_{I}=1$ ). A significant increase (resp. decrease) of $E_{L}$ (resp. $E_{T}$ ) with the increase of $a_{I}$ was observed (Fig. 7a), whereas the shear modulus $G_{L T}$ slightly decreased (Fig. 7b). In contrast, the Poisson ratios followed opposite evolutions: $v_{T L}$ significantly increased from 0.33 up to values larger than 1 with increasing $a_{I}$. The Poisson ratio $v_{L T}$ decreased progressively from 0.33 up to 0 as the fibre orientation intensity $a_{I}$ increased (Fig. 7c). Similar tendencies were observed by Heyden and Gustafsson for fibrous networks with comparable fibre orientation distribution functions (Heyden 2000) (Heyden and Gustafsson 1998).

\section{2 - Comparison between discrete element simulation and the analytical model}

Fig. 8 reveals that the analytical expressions Eqs. (21) and (23) fit well the trends gained from the discrete element simulation when the same microstructure and micromechanical parameters are used as input. Hence, the analytical estimates can be considered as accurate enough predictors of the in-plane elasticity of low-density papers. In particular, this figure shows the relevance of the very simple and compact form Eq. (23) proposed for slender and highly connected fibrous networks.

\section{3 - Comparison with experimental results}

Thanks to the measurements performed with 3D tomographic images (see section 3), all input and microscale parameters of the models could be obtained except the specific shear bond stiffness $\bar{k}_{b}$. This unique unknown parameter was determined to fit the experimental results obtained from tensile tests: $\bar{k}_{b}=2000 \mathrm{~N} \mathrm{~mm}^{-3}$. This is illustrated in Fig. 9, from which several important remarks can be drawn:

- The proposed analytical predictor Eq. (23) allows nice quantitative predictions of the measured Young's moduli $E, E_{L}, E_{T}$, for a wide range of fibre contents, i.e., up to 0.35, and for two different fibre orientation distributions. In particular, the orthotropy was well-captured for the orientated papers, as well as the non-linearity of the Young's moduli with the fibre content $\phi$. According to the assumptions stated to build the model, the last trend may be ascribed to three important microstructure parameters, as emphasised from $\mathrm{Eq}(16)$ : (i) the number of fibres per unit of volume $n=4 \phi /(\bar{w} \bar{t} \bar{l})$ which is proportional to $\phi$, (ii) the mean coordination number $\bar{z}$ which is also proportional to $\phi$ (Eq. (2)), and (iii) the square of the mean bonding ratio $\bar{r}_{b}^{2}$ where $\bar{r}_{b}$ is an affine function of $\phi$ (Eq. (1)), for the studied low-density papers. Hence, these predictions tend to prove, at least within this concentration regime, that the number, the geometry as well as the stiffness of fibre bond zones should play a leading role on the elasticity of papers.

- A departure of the model prediction from the experimental data is observed above a fibre content of 0.35 , the model overestimating the last ones. For example, at a fibre content of 0.45 , the tensile modulus of planar random papers is close to $5.5 \mathrm{GPa}$, whereas the model prediction is close to $6.5 \mathrm{GPa}$. Further increasing the paper density 
above this concentration would definitely conduct to unrealistic stiff estimations. The first reason to explain this departure is related to the relevance of Eq. (1) at concentration higher than those studied herein: the bonding ratio should probably deviate from the reported linear increase to reach a yield: additional bond scale experimental data would reinforce this possible assumption. The second important reason may be correlated to the increase of the fibre deformation mechanisms far from bonding zones such as tension, compression and shear. This has been highlighted by the numerical simulation of Brandberg and Kulachenko (2017) for high-density papers with increasing bond stiffnesses: compared with low-density papers with low bond stiffnesses (such as the papers studied here), the aforementioned mechanisms are expected to take the leading role (up to $80 \%$ of the total elastic energy). Under such circumstances, the evolution of the elastic moduli with the fibre content should become linear, as already predicted by the previous analytical literature models (see Fig. 5): the proposed model should be improved and modified purposely. 
(a)

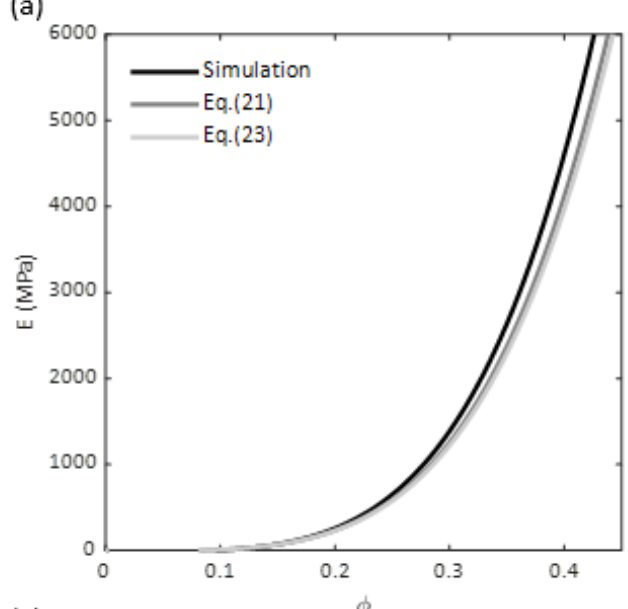

(c)

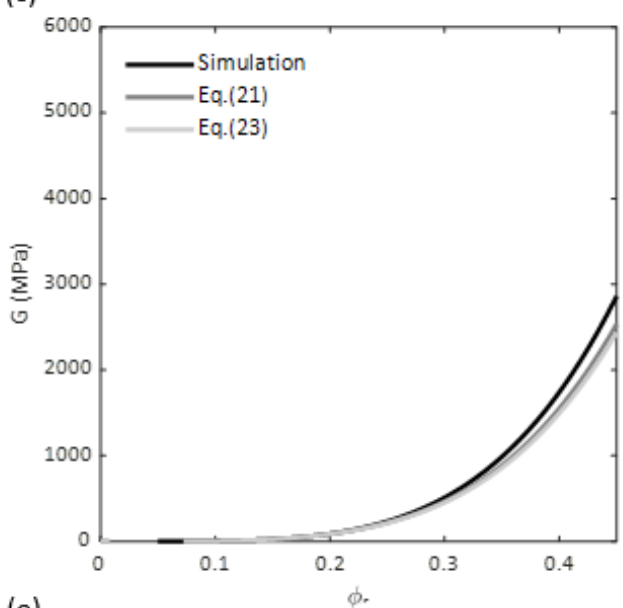

(e)

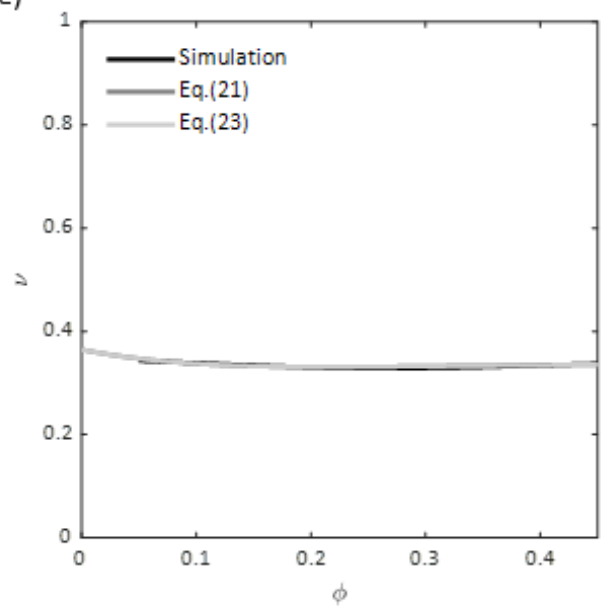

(b)

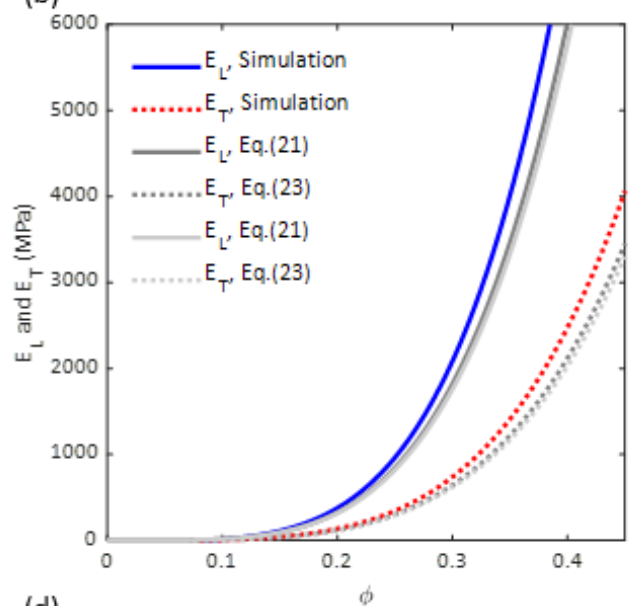

(d)

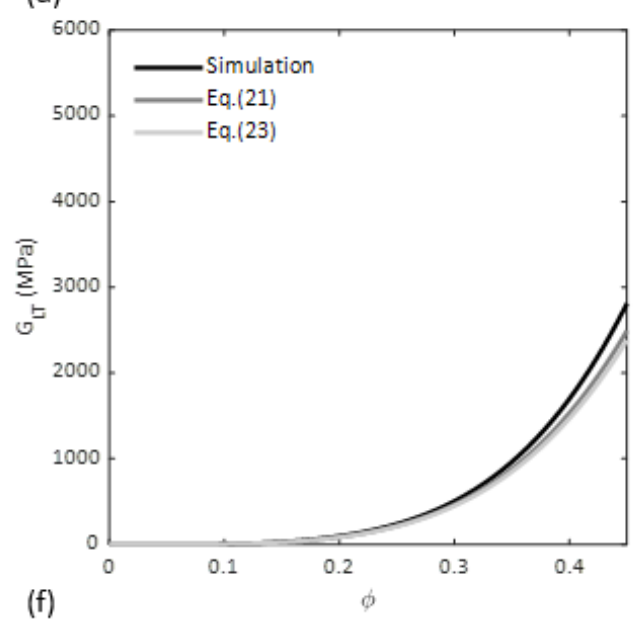

(f)

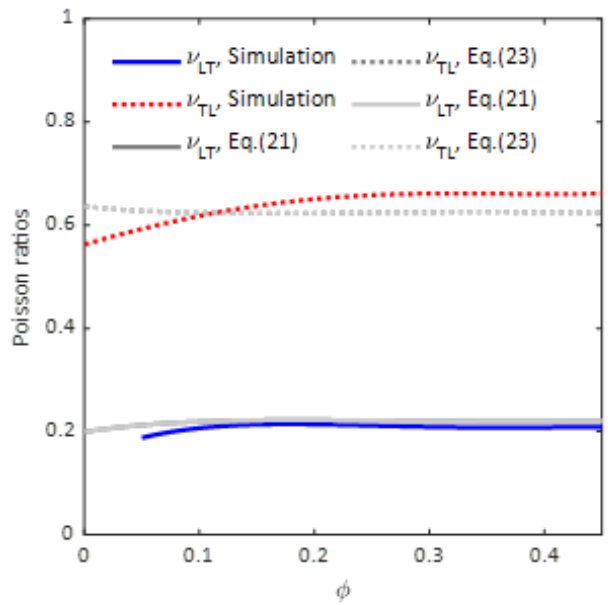

Figure 8. Comparison between the discrete element simulations (black lines) and the proposed analytical models for networks with a planar random fibre orientation $(a, c, e)$ and a preferred moderate fibre alignment along the $\boldsymbol{e}_{1}$-direction (b,d,f), similar to that reported in Figs. 2c-d. For the sake of clarity, only polynomial fits of the numerical results are shown in these figures.

- The relevance of the specific shear bond stiffness value was first investigated in regards of the energy per unit surface area $e_{b}$ which is required to break fibre bonds. By assuming as a first rough but rational approximation that the early stages of plastic deformation in papers, i.e., typically at a macroscopic tensile yield strain $\varepsilon_{Y}$ that ranges 
between 0.2 and $0.5 \%$, mainly arise from the elastic decohesion of fibre bonds, it is possible to get an estimate of $e_{b}$ from the proposed network approach:

$$
e_{b} \approx \frac{1}{2} \bar{k}_{b} \Delta \bar{u}^{2}
$$

where $\Delta \bar{u}$ is the mean relative displacement in the bond regions between bonded fibres (Martoïa et al., 2016a). The displacement $\Delta \bar{u}$ can be estimated with Eq. (20), i.e., $\Delta \bar{u} \approx \varepsilon_{Y} \bar{l} / 4$, so that Eq. (24) should conduct to fibre bond breakage energy $e_{b}$ that should range between $2.510^{-15}$ and $6.2510^{-15} \mathrm{~kJ} \mathrm{um}^{-2}$ (using the estimated value of $\bar{k}_{b}$ ). It must be emphasized that this estimate depends on the area of the bond surface. By performing micromechanical tests on bonded cellulose fibres, several authors showed that $e_{b}$ should range between $10^{-16}$ and $10^{-15} \mathrm{~kJ}_{1 \mathrm{~m}^{-2}}$ (Schmied et al., 2012) (Schmied et al., 2013) (Hirn and Schennach 2015). Considering the uncertainty of our own estimate of the bond surface area, this practically falls into the aforementioned range. Thus, it can be considered that the estimate of the specific bond stiffness $\bar{k}_{b}$ is relevant.

- It is also worth noting that the value of the specific shear bond stiffness $\bar{k}_{b}$ is close to that used in the 3D fibre network simulations performed by Heyden and Gustafsson for cellulosic fibrous materials (Heyden and Gustafsson 1998) (Heyden 2000), i.e., $\bar{k}_{b}=$ $3000 \mathrm{~N} \mathrm{~mm}^{-3}$. In addition, the value of $\bar{k}_{b}$ is rather close, albeit approximately ten times weaker, to the lowest value considered in the fibre network simulation of Brandberg and Kulachenko (2017). In this numerical work, the tangent bond stiffness $\bar{K}_{b}=\bar{k}_{b} \bar{r}_{b}^{2} \bar{w}^{2}$ used for network simulation was deduced from a former numerical study achieved at the bond scale. Fibres with "perfect" geometry were used for that purpose, i.e., very different from the complex ones shown in Fig. 3. Thus, these numerical values should be overvalued due to a possible overestimation of the bonding degree ratio $\bar{r}_{b}$, compared with those that were measured from the $3 \mathrm{D}$ images with real fibres that are wavy and twisted. However, it is interesting to note that the overall bond energy should be the leading deformation mechanism, i.e., with more than $40 \%$ of the overall deformation energy, when the lowest value of $\bar{K}_{b}$ proposed by Brandberg and Kulachenko (2017) is used and when low-density papers are considered. If we extrapolate the results of Brandberg and Kulachenko (2017) to a bond energy ten times lower, i.e., in accordance to our value which is in-line with the experimental results of the literature (see previous point), such a bond contribution is expected to raise up to more than $80 \%$. This reinforces the basic micro-mechanical assumption of the proposed model. 
(a)

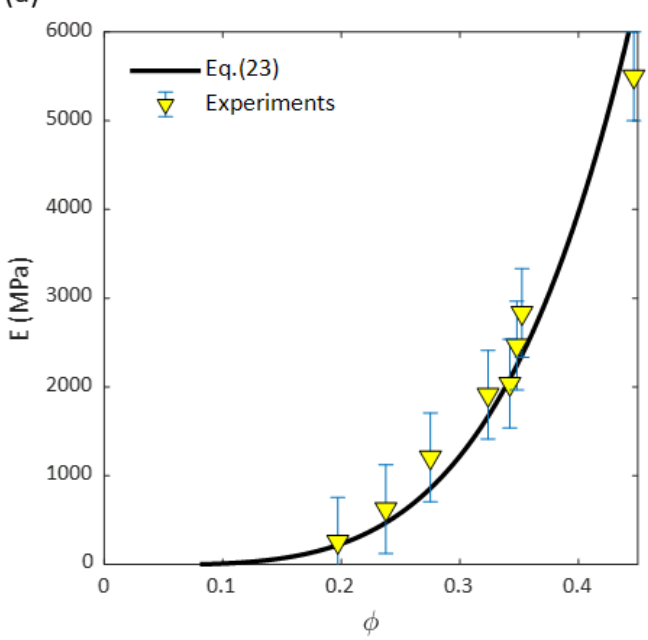

(b)

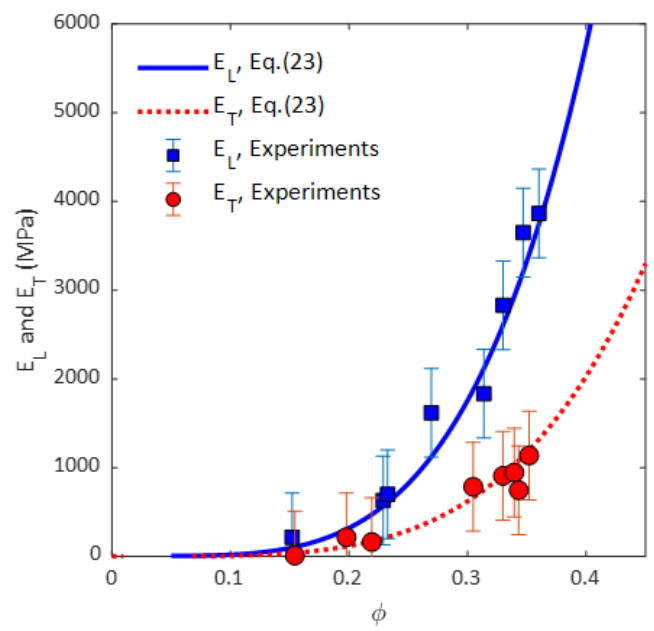

Figure 9. Evolution of the Young's moduli $E, E_{L}, E_{T}$ with the fibre content $\phi$ for papers with planar random fibre orientation (a) and a preferred fibre orientation along the $\boldsymbol{e}_{1}$-direction (b) similar to that reported in Figs. 2c-d: comparison between the predictions of the analytical model Eq. (23) (lines) and the data obtained from tensile tests (symbols). These predictions were obtained using a specific shear bond stiffness $\bar{k}_{b}$ of $2000 \mathrm{~N} \mathrm{~mm}^{-3}$.

\section{6 - Conclusion}

The objective of this study was to link the macroscale elastic properties of low-density paper materials with their complex fibrous microstructures and the related fibre scale deformation mechanisms. For that purpose, model papers with simple planar random and orientated fibrous microstructures were fabricated. Tensile tests showed that their elastic moduli exhibited wellknown planar orthotropy that depended on the fibre orientation. In addition, regardless of the considered fibre orientation, the Young's moduli of papers increased as a power-law function of the fibre content $\phi$ with a power-law exponent close to 4 , which is a trend not observed for papers with higher densities. From the fibre scale 3D images of their microstructures, we also showed that the connectivity of the studied low-density papers was high, i.e., the distance between two adjacent bonds on a fibre was found to be of the same order of magnitude as the fibre width. The fibre connectivity is a function of the geometry, content and orientation of fibres. In addition, a modified expression of the statistical tube model was shown to reasonably predict the fibre connectivity. The 3D images also demonstrated that the mean bonding degree ratio increased linearly with the fibre content but with noticeable low values, i.e., with bonding surface areas much lower than that available for a full fibre coverage (only 1 to $25 \%$ of the latter).

From these experimental data obtained at the fibre scale, fibre network models were proposed to predict the in-plane elastic properties of the considered low-density papers. In these models, fibres were regarded as rigid rods so that the elastic deformation of papers was mainly attributed to the deformation of the fibre bonds and their vicinities. Hence, two macroscale models were established. The first one used fibre scale discrete element simulations and the second was analytical. The latter can be easily used for engineering purposes, e.g., to design novel paper-based applications or to optimise some of the papermaking operations. Both models emphasised the leading roles of the fibre content, orientation and geometry on the macroscale 
elastic properties of the considered papers. Despite its simplicity, the analytical model quantitatively well described trends predicted by the numerical model. If relevant microstructure descriptors are provided as inputs, we have also shown that the prediction of the numerical and analytical models can nicely estimate the elastic properties of the planar random and orientated model low-density papers, for a wide range of fibre contents and with only one unknown parameter, namely, the specific shear bond stiffness. The identified value of the specific shear bond stiffness is in accordance with literature data. Hence, the micromechanical approach developed in this study proves that the fibres bonds, i.e., their number, their geometry as well as their stiffness, play a leading role on the elasticity of lowdensity papers. At higher fibre contents, other leading deformation micro-mechanisms arise, such as tension and shear far from bonding zones, thus inducing a switch from the non-linear dependence of the Young moduli with the fibre content to a linear one. The developed model should be modified accordingly for this fibre content range.

\section{Appendix 1}

In the theory developed by Cox, the Young's modulus $E_{C o x}$ of planar random fibre networks is:

$E_{\text {Cox }}=\frac{1}{3} E_{f} \phi$

where $E_{f}$ is the Young's modulus of the fibres.

In the approach developed by Page and Seth, the Young's modulus $E_{P S}$ of planar random fibre networks is:

$E_{P S}=\frac{1}{3} E_{f} \phi\left[1-\frac{1}{r_{1} R B A} \sqrt{\frac{E_{f}}{2 G_{f}}}\right]$

where $G_{f}$ is the shear modulus of fibres, $r_{1}=l / \bar{w}$ is the fibre slenderness and RBA is the relative bonded area that can be measured using various experimental techniques or estimated analytically using the Sampson's model as follows:

$R B A \approx\left[1-\frac{1}{\bar{c}}\right]\left[1+\frac{(1-\phi)((2-(2+\phi)(1-\phi))}{\ln (1-\phi)}\right]$

where $\bar{c}=\bar{w} G / \delta$ and $\delta=1.5 \times 10^{-4} \mathrm{~g} \mathrm{~m}^{-1}$.

In the 2D network model proposed by Wu and Dzenis, the effective Young's modulus $E_{W D}$ and the Poisson ratio $v_{W D}$ of planar random fibre network are written as a function of the dimensionless fibre density $q$ (for cylindrical fibres of mean diameter $\bar{w}, n=4 \phi r_{1} / \pi$ is the number of fibre per unit of volume) as follows:

$E_{W D}=\frac{\pi E_{f}\left(1-v_{W D}^{2}\right)}{2 r_{1}} n\left\{\frac{3}{16}+2\left[n^{2}\left(\frac{1}{\pi}+\frac{1+\frac{2}{\pi}}{2 r_{1}}\right)^{2}-\frac{1}{4}\right]\left(\frac{a}{1+v_{f}}+b\right)\right\}\left[1-\exp \left(\frac{1-n / n_{c}}{2}\right)\right]$ 
and

$v_{W D}=\frac{\pi^{2}-32\left\{n^{2}\left[\frac{1}{\pi}+\left(1+\frac{2}{\pi}\right) \frac{1}{r_{1}}\right]-\frac{1}{4}\right\}\left[\frac{a}{1+v_{f}}+b\right]}{3 \pi^{2}+32\left\{n^{2}\left[\frac{1}{\pi}+\left(1+\frac{2}{\pi}\right) \frac{1}{r_{1}}\right]-\frac{1}{4}\right\}\left[\frac{a}{1+v_{f}}+b\right]}$,

where $v_{f}$ is the Poisson ratio of the fibres, $n_{c}$ is a percolation threshold beyond which fibres form fully connected planar networks without disjointed substructures (for planar networks with random orientations, some numerical studies showed that $n_{c} \approx 5.7$ (Åström et al., 2000) (Alava and Niskanen 2006)), and $a$ and $b$ are dimensionless numbers that are expressed as a function of both the coordination number $\bar{z}$ and a critical fibre segment length $l_{c} \approx$ $\bar{w} / 2 \sqrt{6\left(1+v_{f}\right)}$ as follows:

$a=\frac{1}{4(\bar{z}+1)^{2}} \int_{0}^{\frac{l(\bar{z}+1)}{l_{c}}} \eta \exp (-\eta) d \eta$

and

$b=\frac{3 \bar{w}^{2}}{8 l^{2}} \int_{\frac{(\bar{z}+1)}{l_{c}}}^{\bar{z}+1} \frac{\exp (-\eta)}{\eta} d \eta$.

\section{Appendix 2}

Accounting for all the assumptions stated in subsection 4.4, the macroscale stress tensor $\boldsymbol{\sigma}$ eqn(11) can be expressed as follows:

$\boldsymbol{\sigma}=\frac{k_{b} \bar{z} \bar{b}^{2} d_{\max }^{2} n}{2 \varphi_{1}}\left(\mathbb{B}_{1}+\mathbb{B}_{2}\right): \boldsymbol{\varepsilon}$

where

$$
\left\{\begin{array}{c}
\mathbb{B}_{1}=\frac{1}{B} \sum_{N} \sum_{\mathcal{B}} s_{i}^{b 2} \mathbf{p}_{i} \otimes \mathbf{p}_{i} \otimes \mathbf{p}_{i} \otimes \mathbf{p}_{i} \\
\mathbb{B}_{2}=-\frac{1}{B} \sum_{N} \sum_{\mathcal{B}} s_{i}^{b} s_{j}^{b} \mathbf{p}_{j} \otimes \mathbf{p}_{i} \otimes \mathbf{p}_{i} \otimes \mathbf{p}_{i}
\end{array}\right.
$$

By assuming that fibre bonds are uniformly distributed along the fibre length, the following discrete sums can be written (Vassal et al., 2008):

$$
\left\{\begin{array}{c}
\sum_{\mathcal{B}} s_{i}^{b 2} \approx \bar{z} \frac{l^{2}}{12}\left(1-\frac{1}{\bar{z}}\right) \\
\sum_{\mathcal{B}} s_{i}^{b} \approx 0
\end{array}\right.
$$

Then, by assuming that $s_{i}$ and $s_{j}$ are uncorrelated, the expressions of $\mathbb{B}_{1}$ and $\mathbb{B}_{2}$ are 


$$
\left\{\begin{array}{c}
\mathbb{B}_{1} \approx \frac{l^{2}}{6}\left(1-\frac{1}{\bar{z}}\right) \mathbb{A} \\
\mathbb{B}_{2} \approx 0
\end{array}\right.
$$

where $\mathbb{A}$ is the fourth-order rod orientation tensor defined in Eq. (22). These two relations were validated using the microstructure generator and yielded to the analytical estimates proposed in Eqs. (21) and (23).

\section{Supplementary information}

(a)

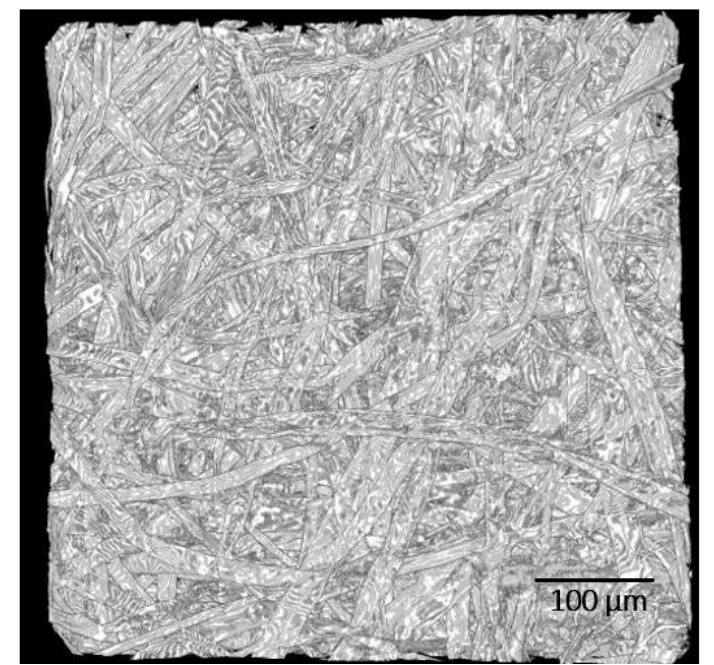

Figure S1. 3D images of model papers of fibre content $\phi=0.323$ with (a) in-plane isotropic and (b) in-plane orientated fibrous microstructures (voxel size: $0 . \gamma^{3} \mu m^{3}$ ). The red arrow shown in image $b$ indicates the preferred fibre orientation.

\section{References}

Advani SG, Tucker CL (1987) The Use of Tensors to Describe and Predict Fiber Orientation in Short Fiber Composites. J Rheol 31:751-784. https://doi.org/10.1122/1.549945

Alava M, Niskanen K (2006) The physics of paper. Rep Prog Phys 69:669-723. https://doi.org/10.1088/0034-4885/69/3/R03

Antoine C, Nygård P, Gregersen $\varnothing \mathrm{W}$, et al (2002) 3D images of paper obtained by phasecontrast X-ray microtomography: image quality and binarisation. Nucl Instrum Methods Phys Res Sect Accel Spectrometers Detect Assoc Equip 490:392-402. https://doi.org/10.1016/S0168-9002(02)01003-3

Åström JA, Mäkinen JP, Alava MJ, Timonen J (2000) Elasticity of Poissonian fiber networks. Phys Rev E 61:5550-5556. https://doi.org/10.1103/PhysRevE.61.5550

Bensoussan A, Lions J-L, Papanicolau G (1978) Asymptotic Analysis for Periodic Structures. North Holland, Amsterdam 
Borodulina S (2016) Extracting fiber and network connectivity data using microtomography images of paper. Nord Pulp Pap Res J 31:469-478

Borodulina S, Motamedian HR, Kulachenko A (2018) Effect of fiber and bond strength variations on the tensile stiffness and strength of fiber networks. Int J Solids Struct 154:19-32. https://doi.org/10.1016/j.ijsolstr.2016.12.013

Brandberg A, Kulachenko A (2017) The effect of geometry changes on the mechanical stiffness of fibre-fibre bonds. In: Advances in Pulp and Paper Research. Manchester, pp 683719 https://doi.org/10.15376/frc.2017.2.683

Brandberg A, Kulachenko A (2020) Compression failure in dense non-woven fiber networks. Cellulose 27:6065-6082. https://doi.org/10.1007/s10570-020-03153-2

Cox HL (1952) The elasticity and strength of paper and other fibrous materials. Br J Appl Phys 3:72-79. https://doi.org/10.1088/0508-3443/3/3/302

DeMaio A, Lowe R, Patterson T, Ragauskas A (2006) Direct observations of bonding influence on the tensile creep behavior of paper. Nord Pulp Pap Res J 21:297-302. https://doi.org/10.3183/npprj-2006-21-03-p297-302

DeMaio A, Patterson T (2008) Similarities in bonding influence between pre-failure tensile creep and stress-strain behavior of paper. Mech Mater 40:133-149. https://doi.org/10.1016/j.mechmat.2007.06.007

Dumont PJJ, Le Corre S, Orgéas L, Favier D (2009) A numerical analysis of the evolution of bundle orientation in concentrated fibre-bundle suspensions. J Non-Newton Fluid Mech 160:76-92. https://doi.org/10.1016/j.jnnfm.2009.03.001

Eder M, Terziev N, Daniel G, Burgert I (2008) The effect of (induced) dislocations on the tensile properties of individual Norway spruce fibres. Holzforschung 62:77-81. https://doi.org/10.1515/HF.2008.011

Fischer WJ, Hirn U, Bauer W, Schennach R (2012) Testing of individual fiber-fiber joints under biaxial load and simultaneous analysis of deformation. Nord Pulp Pap Res J 27:237-244. https://doi.org/10.3183/npprj-2012-27-02-p237-244

Godinho PMJS, Jajcinovic M, Wagner L, et al (2019) A continuum micromechanics approach to the elasticity and strength of planar fiber networks: Theory and application to paper sheets. Eur J Mech ASolids 75:516-531. https://doi.org/10.1016/j.euromechsol.2018.10.005

Gregersen O, Houen PJ, Helle T, et al (2001) Three-Dimensional lmaging of Paper by Use of Synchrotron X-Ray Microtomography. J Pulp Pap Sci 27:50-53

Guiraud O, Orgéas L, Dumont PJJ, Rolland du Roscoat S (2012) Microstructure and deformation micromechanisms of concentrated fiber bundle suspensions: An analysis 
combining x-ray microtomography and pull-out tests. J Rheol 56:593-623. https://doi.org/10.1122/1.3698185

Heyden S (2000) Network modelling for the evaluation of mechanical properties of cellulose fibre fluff. PhD Thesis, Lund University.

Heyden S, Gustafsson P-J (1998) Simulation of Fracture in a Cellulose Fibre Network. J Pulp Pap Sci 24:160-165

Hirn U, Schennach R (2015) Comprehensive analysis of individual pulp fiber bonds quantifies the mechanisms of fiber bonding in paper. Sci Rep 5:10503. https://doi.org/10.1038/srep10503

Jabbour L, Bongiovanni R, Chaussy D, et al (2013) Cellulose-based Li-ion batteries: a review. Cellulose 20:1523-1545. https://doi.org/10.1007/s10570-013-9973-8

Jajcinovic M, Fischer WJ, Hirn U, Bauer W (2016) Strength of individual hardwood fibres and fibre to fibre joints. Cellulose 23:2049-2060. https://doi.org/10.1007/s10570-016-08950

Krasnoshlyk V, Rolland du Roscoat S, Dumont PJJ, et al (2018) Three-dimensional visualization and quantification of the fracture mechanisms in sparse fibre networks using multiscale X-ray microtomography. Proc R Soc Math Phys Eng Sci 474:20180175. https://doi.org/10.1098/rspa.2018.0175

Kulachenko A, Denoyelle T, Galland S, Lindström SB (2012) Elastic properties of cellulose nanopaper. Cellulose 19:793-807. https://doi.org/10.1007/s10570-012-9685-5

Kulachenko A, Uesaka T (2012) Direct simulations of fiber network deformation and failure. Mech Mater 51:1-14

Le Corre S, Caillerie D, Orgéas L, Favier D (2004) Behavior of a net of fibers linked by viscous interactions: theory and mechanical properties. J Mech Phys Solids 52:395-421. https://doi.org/10.1016/S0022-5096(03)00090-5

Le Corre S, Dumont P, Orgéas L, Favier D (2005) Rheology of highly concentrated planar fiber suspensions. J Rheol 49:1029-1058. https://doi.org/10.1122/1.1993594

Macfarlane AL, Kadla JF, Kerekes RJ (2012) High Performance Air Filters Produced from Freeze-Dried Fibrillated Wood Pulp: Fiber Network Compression Due to the Freezing Process. Ind Eng Chem Res 51:10702-10711. https://doi.org/10.1021/ie301340q

Magnusson MS (2016) Investigation of interfibre joint failure and how to tailor their properties for paper strength. Nord Pulp Pap Res J 31:109-122. https://doi.org/10.3183/npprj2016-31-01-p109-122 
Mansour R, Kulachenko A, Chen W and Olsson M (2019) Stochastic Constitutive Model of Isotropic Thin Fiber Networks Based on Stochastic Volume Elements, Materials, 12, 538, http://dx.doi.org/10.3390/ma12030538

Martoïa F, Dumont PJJ, Orgéas L, et al (2016a) On the origins of the elasticity of cellulose nanofiber nanocomposites and nanopapers: a micromechanical approach. RSC Adv 6:47258-47271. https://doi.org/10.1039/C6RA07176G

Martoïa F, Dumont PJJ, Orgéas L, et al (2016b) Micro-mechanics of electrostatically stabilized suspensions of cellulose nanofibrils under steady state shear flow. Soft Matter 12:17211735. https://doi.org/10.1039/C5SM02310F

Martoïa F, Orgéas L, Dumont PJJ, et al (2017) Crumpled paper sheets: Low-cost biobased cellular materials for structural applications. Mater Des 136:150-164. https://doi.org/10.1016/j.matdes.2017.09.031

Marulier C, Dumont PJJ, Orgéas L, et al (2012) Towards 3D analysis of pulp fibre networks at the fibre and bond levels. Nord Pulp Pap Res J 27:245

Marulier C, Dumont PJJ, Orgéas L, et al (2015) 3D analysis of paper microstructures at the scale of fibres and bonds. Cellulose 22:1517-1539. https://doi.org/10.1007/s10570-0150610-6

Motamedian HR, Halilovic AE, Kulachenko A (2019) Mechanisms of strength and stiffness improvement of paper after PFI refining with a focus on the effect of fines. Cellulose 26:4099-4124. https://doi.org/10.1007/s10570-019-02349-5

Motamedian HR, Kulachenko A (2019) Simulating the hygroexpansion of paper using a 3D beam network model and concurrent multiscale approach. Int J Solids Struct 161:2341. https://doi.org/10.1016/j.ijsolstr.2018.11.006

Nanko H, Ohsawa (1989) Mechanisms of fibre bond formation. Fundam. Papermak. Trans IXth Fund Res Symp Camb. 783-830

Neagu RC, Gamstedt EK, Berthold F (2006) Stiffness Contribution of Various Wood Fibers to Composite Materials, J Compos Mater, 40, 663, https://doi.org/10.1177/0021998305055276

Niskanen K (2011) Mechanics of Paper Products. Walter de Gruyter

Niskanen K, Krenlampi P (1998) In-plane tensile properties. In: Papermaking science and technology, paper physics, Fapet Oy, Helsinki

Nyholm K, Ander P, Bardage S, Geoffrey D (2001) Dislocations in pulp fibres - their origin, characteristics and importance - a review. Nord Pulp Pap Res J 16:376-384. https://doi.org/10.3183/npprj-2001-16-04-p376-384 
Oyola-Reynoso S, Heim AP, Halbertsma-Black J, et al (2015) Draw your assay: Fabrication of low-cost paper-based diagnostic and multi-well test zones by drawing on a paper. Talanta 144:289-293. https://doi.org/10.1016/j.talanta.2015.06.018

Page DH, Seth RS (1980) The elastic modulus of paper. II: The importance of fiber modulus, bonding, and fiber length. Tappi 63:113-116

Page DH, Seth RS, DeGrace JH (1979) The Elastic Modulus of Paper - The Controlling Mechanisms. Tappi 62:99-102

Page DH, Tydeman PA, Hunt M (1962) A Study of Fibre-to-Fibre Bonding by Direct Observation. Form Struct Pap 1:171-194

Park NA, Ko YC, Melani L and Kim HJ (2020) Mechanical properties of low-density paper. Nordic Pulp Paper J, Nordic Pulp Paper Res J 2020; 35(1): 61-70 https://doi.org/10.1515/npprj-2019-0052

Räisänen VI, Alava MJ, Niskanen KJ, Nieminen RM (1997) Does the shear-lag model apply to random fiber networks? J Mater Res 12:2725-2732. https://doi.org/10.1557/JMR.1997.0363

M. Rigdahl, H. Andersson, B. Westerlind and H. Hollmark (1983) Elastic Behaviour of Low Density Paper Described by Network Mechanics. Fibre Sci Technol 19, 127

Rolland du Roscoat S, Bloch J-F, Thibault X (2005) Synchrotron radiation microtomography applied to investigation of paper. J Phys Appl Phys 38:A78-A84. https://doi.org/10.1088/0022-3727/38/10A/015

Rolland du Roscoat S, Decain M, Thibault X, et al (2007) Estimation of microstructural properties from synchrotron X-ray microtomography and determination of the REV in paper materials. Acta $\quad$ 5ater 2841-2850. https://doi.org/10.1016/j.actamat.2006.11.050

Salmen L, Fellers C (1989) The nature of volume hydroexpansivity of paper. Nat Vol Hydroexpansivity Pap 15:J63-J65

Sampson WW (2004) A model for fibre contact in planar random fibre networks. J Mater Sci 39:2775-2781. https://doi.org/10.1023/B:JMSC.0000021453.00080.5a

Sampson WW (2008) Modelling Stochastic Fibrous Materials with Mathematica®. Springer Science \& Business Media

Sanchez-Palencia E (1980) Non-Homogeneous Media and Vibration Theory. Lecture Notes in Physics. Springer, Berlin, Germany

Schmied FJ, Teichert C, Kappel L, et al (2012) Joint strength measurements of individual fiber-fiber bonds: an atomic force microscopy based method. Rev Sci Instrum 83:073902 
Schmied FJ, Teichert C, Kappel L, et al (2013) What holds paper together: Nanometre scale exploration of bonding between paper fibres. Sci Rep 3:2432. https://doi.org/10.1038/srep02432

Schulgasser K (1981) On the in-plane elastic constants of paper. Fibre Sci Technol 15:257-270. https://doi.org/10.1016/0015-0568(81)90051-8

Schulgasser K, Page DH (1988) The influence of tranverse fibre properties on the in-plane elastic behaviour of paper. Compos Sci Technol 32:279-292

Simon J-W (2020) A Review of Recent Trends and Challenges in Computational Modeling of Paper and Paperboard at Different Scales. Arch Comput Methods Eng. https://doi.org/10.1007/s11831-020-09460-y

Toll S (1993) Note: On the tube model for fiber suspensions. J Rheol 37:123-125. https://doi.org/10.1122/1.550460

Tollenaere H, Caillerie D (1998) Continuous modeling of lattice structures by homogenization. Adv Eng Softw 29:699-705. https://doi.org/10.1016/S0965-9978(98)00034-9

Twede D, Selke SE, Kamdem D-P, Shires D (2014) Cartons, crates and corrugated board: handbook of paper and wood packaging technology. DEStech Publications, Inc

Vassal J-P, Orgéas L, Favier D, et al (2008) Upscaling the diffusion equations in particulate media made of highly conductive particles. II. Application to fibrous materials. Phys Rev E 77:011303. https://doi.org/10.1103/PhysRevE.77.011303

Viguié J, Dumont PJJ, Orgéas L, et al (2011) Surface stress and strain fields on compressed panels of corrugated board boxes. An experimental analysis by using Digital Image Stereocorrelation. Compos Struct 93:2861-2873. https://doi.org/10.1016/j.compstruct.2011.05.018

Viguié J, Latil P, Orgéas L, et al (2013) Finding fibres and their contacts within 3D images of disordered fibrous media. Compos Sci Technol 89:202-210. https://doi.org/10.1016/j.compscitech.2013.09.023

Wernersson EL, Borgefors G, Borodulina S, Kulachenko A (2014) Characterisations of fibre networks in paper using micro computed tomography images. Nord Pulp Pap Res J $29: 468-475$

Wu X-F, Dzenis YA (2005) Elasticity of planar fiber networks. J Appl Phys 98:093501. https://doi.org/10.1063/1.2123369²

\section{$\underline{\text { Declarations }}$}

\section{Acknowledgements}


The 3SR Lab and the LGP2 are part of the LabEx Tec 21 (Investissements d'Avenir: grant agreement no. ANR-11-LABX-0030) and the PolyNat Carnot Institute (Investissements d'Avenir: grant agreement no. ANR16-CARN-0025-01).

\section{Funding}

C.M wish to thank Univ. Grenoble Alpes for its $\mathrm{PhD}$ research grant. No other financial support was received for the submitted work.

\section{Availability of data and material}

Yes

Code availability (software application or custom code)

Not applicable

\section{Compliance with ethical standars - Conflicts of interest}

The authors declare they have no conflict of interest

\section{Compliance with ethical standars - Financial interest}

The authors declare they have no financial interests

\section{Compliance with ethical standars - Living participants}

The authors declare that the submitted work involved no Human Participant and Animals.

\section{Compliance with ethical standars - Consent}

All the authors consent and are informed that this work is submitted in the journal Cellulose.

\section{Compliance with ethical standars - Originality}

The authors declare that this work is original and has not been submitted as a research paper in antoher journal. 